\title{
Boundary Harnack principle and Martin boundary at infinity for subordinate Brownian motions
}

\author{
Panki Kim* Renming Song ${ }^{\dagger}$ and Zoran Vondraček ${ }^{\ddagger}$
}

\begin{abstract}
In this paper we study the Martin boundary of unbounded open sets at infinity for a large class of subordinate Brownian motions. We first prove that, for such subordinate Brownian motions, the uniform boundary Harnack principle at infinity holds for arbitrary unbounded open sets. Then we introduce the notion of $\kappa$-fatness at infinity for open sets and show that the Martin boundary at infinity of any such open set consists of exactly one point and that point is a minimal Martin boundary point.
\end{abstract}

AMS 2010 Mathematics Subject Classification: Primary 60J45, Secondary 60J25, 60J50.

Keywords and phrases: Lévy processes, subordinate Brownian motion, harmonic functions, boundary Harnack principle, Martin kernel, Martin boundary, Poisson kernel

\section{Introduction and main results}

The study of the boundary Harnack principle for non-local operators started in the late 1990's with [2] which proved that the boundary Harnack principle holds for the fractional Laplacian (or equivalently the rotationally invariant stable process) in bounded Lipschitz domains. This boundary Harnack principle was extended to arbitrary open sets in [22]. The final word in the case of the rotationally invariant $\alpha$-stable process was given in [4 where the so called uniform boundary Harnack principle was proved in arbitrary open sets with a constant not depending on the set itself. Subsequently, the boundary Harnack principle was extended to more general symmetric Lévy processes, more precisely to subordinate Brownian motions with ever more weaker assumptions on the Laplace exponents of the subordinators, see [10], [12], [13] and [9]. Recently in [5], a boundary Harnack principle was established in the setting of jump processes in metric measure spaces.

Let us be more specific and state the (slightly stronger) assumptions under which the boundary Harnack principle was proved in [13]. Let $S=\left(S_{t}\right)_{t \geq 0}$ be a subordinator (a nonnegative Lévy

${ }^{*}$ This work was supported by Basic Science Research Program through the National Research Foundation of Korea(NRF) grant funded by the Korea government(MEST) (2012-0000940).

${ }^{\dagger}$ Research supported in part by a grant from the Simons Foundation (208236).

${ }^{\ddagger}$ Supported in part by the MZOS grant 037-0372790-2801. 
process with $\left.S_{0}=0\right)$ with Laplace exponent $\phi$ and $W=\left(W_{t}, \mathbb{P}_{x}\right)_{t \geq 0, x \in \mathbb{R}^{d}}$ be a Brownian motion in $\mathbb{R}^{d}, d \geq 1$, independent of $S$ with

$$
\mathbb{E}_{x}\left[e^{i \xi \cdot\left(W_{t}-W_{0}\right)}\right]=e^{-t|\xi|^{2}} \quad \xi \in \mathbb{R}^{d}, t>0
$$

The process $X=\left(X_{t}, \mathbb{P}_{x}\right)_{t \geq 0, x \in \mathbb{R}^{d}}$ defined by $X_{t}:=W\left(S_{t}\right)$ is called a subordinate Brownian motion. It is a rotationally invariant Lévy process in $\mathbb{R}^{d}$ with characteristic exponent $\phi\left(|\xi|^{2}\right)$ and infinitesimal generator $-\phi(-\Delta)$. Here $\Delta$ denotes the Laplacian and $\phi(-\Delta)$ is defined through functional calculus.

The function $\phi$ is a Bernstein function having the representation

$$
\phi(\lambda)=a+b \lambda+\int_{(0, \infty)}\left(1-e^{-\lambda t}\right) \mu(d t)
$$

where $a, b \geq 0$ and $\mu$ is the measure satisfying $\int_{(0, \infty)}(1 \wedge t) \mu(d t)<\infty$, called the Lévy measure of $\phi$ (or $S$ ). Recall that $\phi$ is a complete Bernstein function if the measure $\mu$ has a completely monotone density. For basic facts about complete Bernstein functions, see [20].

Let us introduce the following upper and lower scaling conditions on $\phi$ at infinity:

(H1): There exist constants $0<\delta_{1} \leq \delta_{2}<1$ and $a_{1}, a_{2}>0$ such that

$$
a_{1} \lambda^{\delta_{1}} \phi(t) \leq \phi(\lambda t) \leq a_{2} \lambda^{\delta_{2}} \phi(t), \quad \lambda \geq 1, t \geq 1
$$

This is a condition on the asymptotic behavior of $\phi$ at infinity and it governs the behavior of the subordinator $S$ for small time and small space (see [13, 15]). Note that it follows from the second inequality above that $\phi$ has no drift, i.e., $b=0$. Suppose that $\phi$ is a complete Bernstein function with the killing term $a=0$ and that (H1) holds. The following boundary Harnack principle is proved in [13, Theorem 1.1]: There exists a constant $c=c(\phi, d)>0$ such that for every $z \in \mathbb{R}^{d}$, every open set $D \subset \mathbb{R}^{d}$, every $r \in(0,1)$ and any nonnegative functions $u, v$ on $\mathbb{R}^{d}$ which are regular harmonic in $D \cap B(z, r)$ with respect to $X$ and vanish in $D^{c} \cap B(z, r)$,

$$
\frac{u(x)}{v(x)} \leq c \frac{u(y)}{v(y)}, \quad x, y \in D \cap B(z, r / 2) .
$$

Here, and in the sequel, $B(z, r)$ denotes the open ball in $\mathbb{R}^{d}$ centered at $z$ with radius $r$. This result was obtained as a simple consequence of the following approximate factorization of nonnegative harmonic functions, see [13, Lemma 5.5]: There exists a constant $c=c(\phi, d)>1$ such that for every $z \in \mathbb{R}^{d}$, every open set $D \subset B(z, r)$ and any nonnegative function $u$ on $\mathbb{R}^{d}$ which is regular harmonic in $D$ with respect to $X$ and vanishes a.e. in $D^{c} \cap B(z, r)$,

$$
c^{-1} \mathbb{E}_{x}\left[\tau_{D}\right] \int_{B(z, r / 2)^{c}} j(|y-z|) u(y) d y \leq u(x) \leq c \mathbb{E}_{x}\left[\tau_{D}\right] \int_{B(z, r / 2)^{c}} j(|y-z|) u(y) d y
$$

for every $x \in D \cap B(z, r / 2)$. Here $j$ denotes the density of the Lévy measure of $X$ and $\tau_{D}$ the first exit time of $X$ from $D$. In the case of the rotationally invariant $\alpha$-stable process, (1.2) is proved earlier in [4]. 
Note that the boundary Harnack principle is a result about the decay of non-negative harmonic functions near the (finite) boundary points. It is an interesting problem to study the decay of nonnegative harmonic functions at infinity (which may be regarded as a "boundary point at infinity" of unbounded sets). This is the main topic of the current paper. In order to study the behavior of harmonic functions at infinity, one needs large space and large time properties of the underlying process $X$. This requires a different type of assumptions than (H1) which gives only small space and small time properties of $X$. Therefore, in addition to (H1), we will assume the corresponding upper and lower scaling conditions of $\phi$ near zero:

(H2): There exist constants $0<\delta_{3} \leq \delta_{4}<1$ and $a_{3}, a_{4}>0$ such that

$$
a_{3} \lambda^{\delta_{4}} \phi(t) \leq \phi(\lambda t) \leq a_{4} \lambda^{\delta_{3}} \phi(t), \quad \lambda \leq 1, t \leq 1
$$

This is a condition on the asymptotic behavior of $\phi$ at zero and it governs the behavior of the subordinator $S$ for large time and large space (see [15] for details and examples).

In the recent preprint [15] we studied the potential theory of subordinate Brownian motions under the assumption that $\phi$ is a complete Bernstein function satisfying both conditions (H1) and (H2). We were able to extend many potential-theoretic results that were proved under (H1) (or similar assumptions on the small time and small space behavior) for radii $r \in(0,1)$ to the case of all $r>0$ (with a uniform constant not depending on $r>0$ ). In particular, we proved a uniform boundary Harnack principle with explicit decay rate (in open sets satisfying the interior and the exterior ball conditions) which is valid for all $r>0$. The current paper is a continuation of [15] and is based on the results of [15].

For any open set $D$, we use $X^{D}$ to denote the subprocess of $X$ killed upon exiting $D$. In case $D$ is a Greenian open set in $\mathbb{R}^{d}$ we will use $G_{D}(x, y)$ to denote the Green function of $X^{D}$. For a Greenian open set $D \subset \mathbb{R}^{d}$, let

$$
K_{D}(x, y):=\int_{D} G_{D}(x, z) j(|z-y|) d z, \quad(x, y) \in D \times \bar{D}^{c}
$$

be the Poisson kernel of $X$ in $D \times \bar{D}^{c}$.

The first goal of this paper is to prove the following approximate factorization of regular harmonic functions vanishing at infinity.

Theorem 1.1 Suppose that $\phi$ is a complete Bernstein function satisfying $(\mathbf{H 1})-(\mathbf{H 2})$, let $d>$ $2\left(\delta_{2} \vee \delta_{4}\right)$, and let $X$ be a rotationally invariant Lévy process in $\mathbb{R}^{d}$ with characteristic exponent $\phi\left(|\xi|^{2}\right)$. For every $a>1$, there exists $C_{1}=C_{1}(\phi, a)>1$ such that for any $r \geq 1$, any open set $U \subset \bar{B}(0, r)^{c}$ and any nonnegative function $u$ on $\mathbb{R}^{d}$ which is regular harmonic with respect to $X$ in $U$ and vanishes a.e. on $\bar{B}(0, r)^{c} \backslash U$, it holds that

$$
C_{1}^{-1} K_{U}(x, 0) \int_{B(0, a r)} u(z) d z \leq u(x) \leq C_{1} K_{U}(x, 0) \int_{B(0, a r)} u(z) d z,
$$

for all $x \in U \cap \bar{B}(0, a r)^{c}$. 
Note that $K_{U}(x, 0)=\int_{U} G_{U}(x, y) j(|y|) d y$. A consequence of the assumption $d>2\left(\delta_{2} \vee \delta_{4}\right)$ (always true for $d \geq 2$ ) in Theorem 1.1 is that the process $X$ is transient and points are polar. Under this assumption, the Green function $G(x, y)$ of the process $X$ exists, and by (2.9) below we have $G_{U}(x, y) \leq G(x, y) \asymp|x-y|^{-d} \phi\left(|x-y|^{-2}\right)^{-1}$. This will be used several times in this paper.

In the case of the rotationally invariant $\alpha$-stable process, Theorem 1.1 (for $a=2$ ) was obtained in [17, Corollary 3] from (1.2) by using the inversion with respect to spheres and the Kelvin transform of harmonic functions for the stable process. Since the Kelvin transform method works only for stable processes we had to use a different approach to prove (1.4). We followed the method used in [13] to prove (1.2), making necessary changes at each step. The main technical difficulty of the proof is the delicate upper estimate of the Poisson kernel $K_{\bar{B}(0, r)^{c}}(x, 0)$ of the complement of the ball given in Lemma 3.2, where the full power of the results from [15] was used.

Theorem 1.1 gives the following scale invariant boundary Harnack inequality at infinity.

Corollary 1.2 (Boundary Harnack Principle at Infinity) Suppose that $\phi$ is a complete Bernstein function satisfying $(\mathbf{H 1})-(\mathbf{H 2}), d>2\left(\delta_{2} \vee \delta_{4}\right)$, and that $X$ is a rotationally invariant Lévy process in $\mathbb{R}^{d}$ with characteristic exponent $\phi\left(|\xi|^{2}\right)$. For each $a>1$ there exists $C_{2}=C_{2}(\phi, a)>1$ such that for any $r \geq 1$, any open set $U \subset \bar{B}(0, r)^{c}$ and any nonnegative functions $u$ and $v$ on $\mathbb{R}^{d}$ that are regular harmonic in $U$ with respect to $X$ and vanish a.e. on $\bar{B}(0, r)^{c} \backslash U$, it holds that

$$
C_{2}^{-1} \frac{u(y)}{v(y)} \leq \frac{u(x)}{v(x)} \leq C_{2} \frac{u(y)}{v(y)}, \quad \text { for all } x, y \in U \cap \bar{B}(0, a r)^{c} .
$$

The boundary Harnack principle is the main tool in identifying the (minimal) Martin boundary (with respect to the process $X$ ) of an open set. Recall that for $\kappa \in(0,1 / 2]$, an open set $D$ is said to be $\kappa$-fat open at $Q \in \partial D$, if there exists $R>0$ such that for each $r \in(0, R)$ there exists a point $A_{r}(Q)$ satisfying $B\left(A_{r}(Q), \kappa r\right) \subset D \cap B(Q, r)$. If $D$ is $\kappa$-fat at each boundary point $Q \in \partial D$ with the same $R>0, D$ is called $\kappa$-fat with characteristics $(R, \kappa)$. In case $X$ is a subordinate Brownian motion via a subordinator with a complete Bernstein Laplace exponent regularly varying at infinity with index in $(0,1)$, it is shown in [10] that the minimal Martin boundary of a bounded $\kappa$-fat open set can be identified with the Euclidean boundary.

Corollary 1.2 enables us to identify the Martin boundary and the minimal Martin boundary at infinity of a large class of open sets with respect to $X$. To be more precise, let us first define $\kappa$-fatness at infinity.

Definition 1.3 Let $\kappa \in(0,1 / 2]$. We say that an open set $D$ in $\mathbb{R}^{d}$ is $\kappa$-fat at infinity if there exists $R>0$ such that for every $r \in[R, \infty)$ there exists $A_{r} \in \mathbb{R}^{d}$ such that $B\left(A_{r}, \kappa r\right) \subset D \cap \bar{B}(0, r)^{c}$ and $\left|A_{r}\right|<\kappa^{-1} r$. The pair $(R, \kappa)$ will be called the characteristics of the $\kappa$-fat open set $D$ at infinity.

Note that all half-space-like open sets, all exterior open sets and all infinite cones are $\kappa$-fat at infinity. Examples of disconnected open sets which are $\kappa$-fat at infinity are 
(i) $\left\{x=\left(x_{1}, \ldots, x_{d-1}, x_{d}\right) \in \mathbb{R}^{d}: x_{d}<0\right.$ or $\left.x_{d}>1\right\}$;

(ii) $\bigcup_{n=1}^{\infty} B\left(x^{(n)}, 2^{n-2}\right)$ with $\left|x^{(n)}\right|=2^{n}$.

Let $D \subset \mathbb{R}^{d}$ be an open set which is $\kappa$-fat at infinity with characteristics $(R, \kappa)$. Fix $x_{0} \in D$ and define

$$
M_{D}(x, y):=\frac{G_{D}(x, y)}{G_{D}\left(x_{0}, y\right)}, \quad x, y \in D, y \neq x_{0}
$$

As the process $X^{D}$ satisfies Hypothesis (B) in [16], $D$ has a Martin boundary $\partial_{M} D$ with respect to $X$ and $M_{D}(x, \cdot)$ is continuously extended to $\partial_{M} D$. A point $w \in \partial_{M} D$ is called an infinite Martin boundary point if every sequence $\left(y_{n}\right)_{n \geq 1}, y_{n} \in D$, converging to $w$ in the Martin topology is unbounded (in the Euclidean metric). The set of all infinite Martin boundary points will be denoted by $\partial_{M}^{\infty} D$ and we call this set the Martin boundary at infinity.

By using the boundary Harnack principle at infinity we first show that if $D$ is $\kappa$-fat at infinity, then there exists the limit

$$
M_{D}(x, \infty)=\lim _{y \in D,|y| \rightarrow \infty} M_{D}(x, y) .
$$

The existence of this limit shows that $\partial_{M}^{\infty} D$ consists of a single point which we denote by $\partial_{\infty}$. Finally, we prove that $\partial_{\infty}$ is a minimal Martin boundary point. These findings are summarized in the second main result of the paper.

Theorem 1.4 Suppose that $\phi$ is a complete Bernstein function satisfying $(\mathbf{H 1})-(\mathbf{H 2}), d>2\left(\delta_{2} \vee\right.$ $\left.\delta_{4}\right)$, and $X$ is a rotationally invariant Lévy process in $\mathbb{R}^{d}$ with characteristic exponent $\phi\left(|\xi|^{2}\right)$. Then the Martin boundary at infinity with respect to $X$ of any open set $D$ which is $\kappa$-fat at infinity consists of exactly one point $\partial_{\infty}$. This point is a minimal Martin boundary point.

We emphasize that this result is proved without any assumption on the finite boundary points. In particular, we do not assume that $D$ is $\kappa$-fat.

To the best of our knowledge, the only case where the Martin boundary at infinity has been identified is the case of the rotationally invariant $\alpha$-stable process, see [4]. Again, the Kelvin transform method was used to transfer results for finite boundary points to the infinite boundary point. As we have already pointed out, the Kelvin transform is not available for more general processes.

We remark here that for one-dimensional Lévy processes (satisfying much weaker assumptions than ours) it is proved in [21, Theorem 4] that the minimal Martin boundary at infinity for the half-line $D=(0, \infty)$ is one point. The question of the Martin boundary at infinity is not addressed in [21].

The paper is organized as follows. In the next section we introduce necessary notation and definitions, and recall some results that follow from (H1) and (H2) obtained in [15]. Section 3 is devoted to the proofs of Theorem 1.1 and Corollary 1.2. At the end of the section we collect some consequences of these two results. In the first part of Section 4 we study non-negative harmonic 
functions in unbounded sets that are $\kappa$-fat at infinity. The main technical result is the oscillation reduction in Lemma 4.7 immediately leading to (1.6). Next we look at the Martin and the minimal Martin boundary at infinity and give a proof of Theorem 1.4. We finish the paper by discussing the Martin boundary of the half-space.

Throughout this paper, the constants $C_{1}, C_{2}, C_{3}, \ldots$ will be fixed. The lowercase constants $c_{1}, c_{2}, \ldots$ will denote generic constants whose exact values are not important and can change from one appearance to another. The dependence of the lower case constants on the dimension $d$ and the function $\phi$ may not be mentioned explicitly. The constant $c$ that depends on the parameters $\delta_{i}$ and $a_{i}, i=1,2,3,4$, appearing in (H1) and (H2) will be simply denoted as $c=c(\phi)$. We will use ":=" to denote a definition, which is read as "is defined to be". For $a, b \in \mathbb{R}, a \wedge b:=\min \{a, b\}$ and $a \vee b:=\max \{a, b\}$.

For any open set $U$, we denote by $\delta_{U}(x)$ the distance between $x$ and the complement of $U$, i.e., $\delta_{U}(x)=\operatorname{dist}\left(x, U^{c}\right)$. For functions $f$ and $g$, the notation " $f \asymp g$ " means that there exist constants $c_{2} \geq c_{1}>0$ such that $c_{1} g \leq f \leq c_{2} g$. For every function $f$, we extend its definition to the cemetery point $\partial$ by setting $f(\partial)=0$. For every function $f$, let $f^{+}:=f \vee 0$. We will use $d x$ to denote the Lebesgue measure in $\mathbb{R}^{d}$ and, for a Borel set $A \subset \mathbb{R}^{d}$, we also use $|A|$ to denote its Lebesgue measure. We denote $\bar{B}(0, r)^{c}:=\left\{y \in \mathbb{R}^{d}:|x-y|>r\right\}$. Finally, for a point $x=\left(x_{1}, \ldots, x_{d-1}, x_{d}\right) \in \mathbb{R}^{d}$ we sometimes write $x=\left(\widetilde{x}, x_{d}\right)$ with $\widetilde{x}=\left(x_{1}, \ldots, x_{d-1}\right) \in \mathbb{R}^{d-1}$.

\section{Preliminaries}

In this section we recall some results from [15]. Recall that a function $\phi:(0, \infty) \rightarrow(0, \infty)$ is a Bernstein function if it is $C^{\infty}$ function on $(0, \infty)$ and $(-1)^{n-1} \phi^{(n)} \geq 0$ for all $n \geq 1$. It is well known that, if $\phi$ is a Bernstein function, then

$$
\phi(\lambda t) \leq \lambda \phi(t) \quad \text { for all } \lambda \geq 1, t>0 .
$$

Clearly (2.1) implies the following observation.

Lemma 2.1 If $\phi$ is a Bernstein function, then every $\lambda>0$,

$$
1 \wedge \lambda \leq \frac{\phi(\lambda t)}{\phi(t)} \leq 1 \vee \lambda, \quad \text { for all } t>0
$$

Note that with this lemma, we can replace expressions of the type $\phi(\lambda t)$, when $\phi$ is a Bernstein function, with $\lambda>0$ fixed and $t>0$ arbitrary, with $\phi(t)$ up to a multiplicative constant depending on $\lambda$. We will often do this without explicitly mentioning it.

Recall that a subordinator $S=\left(S_{t}\right)_{t \geq 0}$ is simply a nonnegative Lévy process with $S_{0}=0$. Let $S=\left(S_{t}\right)_{t \geq 0}$ be a subordinator with Laplace exponent $\phi$. The function $\phi$ is a Bernstein function with $\phi(0)=0$ so it has the representation

$$
\phi(\lambda)=b \lambda+\int_{(0, \infty)}\left(1-e^{-\lambda t}\right) \mu(d t),
$$


where $b \geq 0$ is the drift and $\mu$ the Lévy measure of $S$.

A Bernstein function $\phi$ is a complete Bernstein function if its Lévy measure $\mu$ has a completely monotone density, which will be denoted by $\mu(t)$. Throughout this paper we assume that $\phi$ is a complete Bernstein function. In this case, the potential measure $U$ of $S$ admits a completely monotone density $u(t)$ (cf. [20]).

Conditions (H1)-(H2) imply that

$$
c^{-1}\left(\frac{R}{r}\right)^{\delta_{1} \wedge \delta_{3}} \leq \frac{\phi(R)}{\phi(r)} \leq c\left(\frac{R}{r}\right)^{\delta_{2} \vee \delta_{4}}, \quad 0<r<R<\infty .
$$

(See [15] for details.) Using (2.2), we have the following result which will be used many times later in the paper. (See the proof of [13, Lemma 4.1] for similar computations.)

Lemma 2.2 ([15]) Assume (H1) and (H2). There exists a constant $c=c(\phi) \geq 1$ such that

$$
\begin{aligned}
\int_{0}^{\lambda^{-1}} \phi\left(r^{-2}\right)^{1 / 2} d r \leq c \lambda^{-1} \phi\left(\lambda^{2}\right)^{1 / 2}, & \text { for all } \lambda>0, \\
\lambda^{2} \int_{0}^{\lambda^{-1}} r \phi\left(r^{-2}\right) d r+\int_{\lambda^{-1}}^{\infty} r^{-1} \phi\left(r^{-2}\right) d r \leq c \phi\left(\lambda^{2}\right), & \text { for all } \lambda>0, \\
c^{-1} \phi\left(\lambda^{2}\right)^{-1} \leq \int_{0}^{\lambda^{-1}} r^{-1} \phi\left(r^{-2}\right)^{-1} d r \leq c \phi\left(\lambda^{2}\right)^{-1}, & \text { for all } \lambda>0 .
\end{aligned}
$$

Recall that $S=\left(S_{t}\right)_{t \geq 0}$ is a subordinator with Laplace exponent $\phi$. Let $W=\left(W_{t}\right)_{t \geq 0}$ be a $d$-dimensional Brownian motion, $d \geq 1$, independent of $S$ and with transition density

$$
q(t, x, y)=(4 \pi t)^{-d / 2} e^{-\frac{|x-y|^{2}}{4 t}}, \quad x, y \in \mathbb{R}^{d}, t>0 .
$$

The process $X=\left(X_{t}\right)_{t \geq 0}$ defined by $X_{t}:=W\left(S_{t}\right)$ is called a subordinate Brownian motion. $X$ is a rotationally invariant Lévy process with characteristic exponent $\phi\left(|\xi|^{2}\right), \xi \in \mathbb{R}^{d}$. Throughout this paper $X$ is always such a subordinate Brownian motion. The Lévy measure of $X$ has a density $J(x)=j(|x|)$ where $j:(0, \infty) \rightarrow(0, \infty)$ is given by

$$
j(r):=\int_{0}^{\infty}(4 \pi t)^{-d / 2} e^{-r^{2} /(4 t)} \mu(t) d t .
$$

Note that $j$ is continuous and decreasing. Recall that the infinitesimal generator $\mathcal{L}$ of the process $X$ (e.g. [19, Theorem 31.5]) is given by

$$
\mathcal{L} f(x)=\int_{\mathbb{R}^{d}}\left(f(x+y)-f(x)-y \cdot \nabla f(x) \mathbf{1}_{\{|y| \leq \varepsilon\}}\right) J(y) d y
$$

for every $\varepsilon>0$ and $f \in C_{b}^{c}\left(\mathbb{R}^{d}\right)$, where $C_{b}^{c}\left(\mathbb{R}^{d}\right)$ is the collection of $C^{2}$ functions which, along with its partial derivatives of up to order 2 , are bounded. 
By the Chung-Fuchs criterion the process $X$ is transient if and only if

$$
\int_{0}^{1} \frac{\lambda^{d / 2-1}}{\phi(\lambda)} d \lambda<\infty .
$$

It follows that $X$ is always transient when $d \geq 3$. In case (H2) holds, $X$ is transient provided $\delta_{4}<d / 2$ (which is true if $d \geq 2$ ). When $X$ is transient the occupation measure of $X$ admits a density $G(x, y)$ which is called the Green function of $X$ and is given by the formula $G(x, y)=g(|x-y|)$ where

$$
g(r):=\int_{0}^{\infty}(4 \pi t)^{-d / 2} e^{-r^{2} /(4 t)} u(t) d t .
$$

Here $u$ is the potential density of the subordinator $S$. Note that by the transience assumption, the integral converges. Moreover, $g$ is continuous and decreasing. Furthermore, (H1)-(H2) imply the following estimates.

Theorem 2.3 ([15]) Assume both (H1) and (H2).

(a) It holds that

$$
j(r) \asymp r^{-d} \phi\left(r^{-2}\right), \quad \text { for all } r>0 .
$$

(b) If $d>2\left(\delta_{2} \vee \delta_{4}\right)$ then the process $X$ is transient and it holds

$$
g(r) \asymp r^{-d} \phi\left(r^{-2}\right)^{-1}, \quad \text { for all } r>0 .
$$

As a consequence of (2.8), we have

Corollary 2.4 Assume $(\mathbf{H 1})$ and $\mathbf{( H 2 ) . ~ F o r ~ e v e r y ~} L>1$, there exists a constant $c=c(L)>0$ such that

$$
j(r) \leq c j(L r), \quad r>0 .
$$

For any open set $D$, we use $\tau_{D}$ to denote the first exit time of $D$, i.e., $\tau_{D}=\inf \left\{t>0: X_{t} \notin D\right\}$. Given an open set $D \subset \mathbb{R}^{d}$, we define $X_{t}^{D}(\omega)=X_{t}(\omega)$ if $t<\tau_{D}(\omega)$ and $X_{t}^{D}(\omega)=\partial$ if $t \geq \tau_{D}(\omega)$, where $\partial$ is a cemetery state.

Let $p(t, x, y)$ be the transition density of $X$. By the strong Markov property,

$$
p_{D}(t, x, y):=p(t, x, y)-\mathbb{E}_{x}\left[p\left(t-\tau_{D}, X_{\tau_{D}}, y\right) ; t>\tau_{D}\right], \quad x, y \in D,
$$

is the transition density of $X^{D}$. A subset $D$ of $\mathbb{R}^{d}$ is said to be Greenian (for $X$ ) if $X^{D}$ is transient. For a Greenian set $D \subset \mathbb{R}^{d}$, let $G_{D}(x, y)$ denote the Green function of $X^{D}$, i.e.,

$$
G_{D}(x, y):=\int_{0}^{\infty} p_{D}(t, x, y) d t \quad \text { for } \quad x, y \in D .
$$

We define the Poisson kernel $K_{D}(x, z)$ of $D$ with respect to $X$ by

$$
K_{D}(x, z)=\int_{\bar{D}^{c}} G_{D}(x, y) J(y, z) d y, \quad(x, z) \in D \times \bar{D}^{c} .
$$


Then by [7, Theorem 1] we get that for every Greenian open subset $D$, every nonnegative Borel measurable function $f \geq 0$ and $x \in D$,

$$
\mathbb{E}_{x}\left[f\left(X_{\tau_{D}}\right) ; X_{\tau_{D^{-}}} \neq X_{\tau_{D}}\right]=\int_{\bar{D}^{c}} K_{D}(x, y) f(y) d y .
$$

Using the continuities of $G_{D}$ and $J$, one can easily check that $K_{D}$ is continuous on $D \times \bar{D}^{c}$.

Equations (2.8) and (2.11) give the following estimates on the Poisson kernel of $B\left(x_{0}, r\right)$ for all $r>0$.

Proposition 2.5 ([15]) Assume (H1) and (H2). There exist $c_{1}=c_{1}(\phi)>0$ and $c_{2}=c_{2}(\phi)>0$ such that for every $r>0$ and $x_{0} \in \mathbb{R}^{d}$,

$$
\begin{aligned}
K_{B\left(x_{0}, r\right)}(x, y) & \leq c_{1} j\left(\left|y-x_{0}\right|-r\right)\left(\phi\left(r^{-2}\right) \phi\left(\left(r-\left|x-x_{0}\right|\right)^{-2}\right)\right)^{-1 / 2} \\
& \leq c_{1} j\left(\left|y-x_{0}\right|-r\right) \phi\left(r^{-2}\right)^{-1}
\end{aligned}
$$

for all $(x, y) \in B\left(x_{0}, r\right) \times{\overline{B\left(x_{0}, r\right)}}^{c}$ and

$$
K_{B\left(x_{0}, r\right)}\left(x_{0}, y\right) \geq c_{2} j\left(\left|y-x_{0}\right|\right) \phi\left(r^{-2}\right)^{-1}, \quad \text { for all } y \in{\overline{B\left(x_{0}, r\right)}}^{c} .
$$

To discuss the Harnack inequality and the boundary Harnack principle, we first recall the definition of harmonic functions.

Definition 2.6 A function $f: \mathbb{R}^{d} \rightarrow[0, \infty)$ is said to be

(1) harmonic in an open set $D \subset \mathbb{R}^{d}$ with respect to $X$ if for every open set $B$ whose closure is a compact subset of $D$,

$$
f(x)=\mathbb{E}_{x}\left[f\left(X\left(\tau_{B}\right)\right)\right] \quad \text { for every } x \in B
$$

(2) regular harmonic in $D$ for $X$ if for each $x \in D, f(x)=\mathbb{E}_{x}\left[f\left(X\left(\tau_{D}\right)\right) ; \tau_{D}<\infty\right]$;

(2) harmonic for $X^{D}$ if it is harmonic for $X$ in $D$ and vanishes outside $D$.

We note that, by the strong Markov property of $X$, every regular harmonic function is automatically harmonic.

Under the assumptions (H1) and (H2), the following uniform Harnack inequality and uniform boundary Harnack principle for all $r>0$ are true.

Theorem 2.7 ([15]) Assume (H1) and (H2). There exists $c=c(\phi)>0$ such that, for any $r>0, x_{0} \in \mathbb{R}^{d}$, and any function $u$ which is nonnegative on $\mathbb{R}^{d}$ and harmonic with respect to $X$ in $B\left(x_{0}, r\right)$, we have

$$
u(x) \leq c u(y), \quad \text { for all } x, y \in B\left(x_{0}, r / 2\right) .
$$


Theorem 2.8 ([15]) Assume (H1) and (H2). There exists a constant $c=c(\phi)>0$ such that for every $z_{0} \in \mathbb{R}^{d}$, every open set $D \subset \mathbb{R}^{d}$, every $r>0$ and any nonnegative functions $u, v$ in $\mathbb{R}^{d}$ which are regular harmonic in $D \cap B\left(z_{0}, r\right)$ with respect to $X$ and vanish in $D^{c} \cap B\left(z_{0}, r\right)$, we have

$$
\frac{u(x)}{v(x)} \leq c \frac{u(y)}{v(y)}, \quad \text { for all } x, y \in D \cap B\left(z_{0}, r / 2\right) .
$$

For $x \in \mathbb{R}^{d}$, let $\delta_{\partial D}(x)$ denote the Euclidean distance between $x$ and $\partial D$. Recall that $\delta_{D}(x)$ is the Euclidean distance between $x$ and $D^{c}$.

We say that an open set $D$ satisfies both the uniform interior ball condition and the uniform exterior ball condition with radius $R$ for every $x \in D$ radius $R$ if for every $x \in D$ with $\delta_{\partial D}(x) \leq R$ and $y \in \mathbb{R}^{d} \backslash \bar{D}$ with $\delta_{\partial D}(y) \leq R$, there are $z_{x}, z_{y} \in \partial D$ so that $\left|x-z_{x}\right|=\delta_{\partial D}(x),\left|y-z_{y}\right|=\delta_{\partial D}(y)$ and that $B\left(x_{0}, R\right) \subset D$ and $B\left(y_{0}, R\right) \subset \mathbb{R}^{d} \backslash \bar{D}$, where $x_{0}=z_{x}+R\left(x-z_{x}\right) /\left|x-z_{x}\right|$ and $y_{0}=$ $z_{y}+R\left(y-z_{y}\right) /\left|y-z_{y}\right|$.

The following is the one of main results in [15] - the global uniform boundary Harnack principle with explicit decay rate on open sets in $\mathbb{R}^{d}$ with the interior and exterior ball conditions with radius $R$ for all $R>0$.

Theorem 2.9 ([15]) Assume (H1) and (H2). There exists $c=c(\phi)>0$ such that for every open set $D$ satisfying the interior and exterior ball conditions with radius $R>0, r \in(0, R]$, every $Q \in \partial D$ and every nonnegative function $u$ in $\mathbb{R}^{d}$ which is harmonic in $D \cap B(Q, r)$ with respect to $X$ and vanishes continuously on $D^{c} \cap B(Q, r)$, we have

$$
\frac{u(x)}{u(y)} \leq c \sqrt{\frac{\phi\left(\delta_{D}(y)^{-2}\right)}{\phi\left(\delta_{D}(x)^{-2}\right)}} \quad \text { for every } x, y \in D \cap B\left(Q, \frac{r}{2}\right) .
$$

\section{Boundary Harnack principle at infinity}

The goal of this section is to prove the scale invariant boundary Harnack principle at infinity (Theorem 1.1 and Corollary 1.2). In the remainder of this paper we assume that $\phi$ is a complete Bernstein function satisfying (H1) $-(\mathbf{H 2})$ and $d>2\left(\delta_{2} \vee \delta_{4}\right)$, and that $X$ is a rotationally invariant Lévy process in $\mathbb{R}^{d}$ with characteristic exponent $\phi\left(|\xi|^{2}\right)$. Under these assumptions, by (2.9), $g$ satisfies the following property which we will use frequently: For every $L>1$, there exists $c=$ $c(L, \phi)>0$ such that

$$
g(r) \leq c g(L r), \quad r>0 .
$$

To prove Theorem 1.1 we need several lemmas. For $x \in \mathbb{R}^{d}$ and $0<r_{1}<r_{2}$, we use $A\left(x, r_{1}, r_{2}\right)$ to denote the annulus $\left\{y \in \mathbb{R}^{d}: r_{1}<|y-x| \leq r_{2}\right\}$.

Lemma 3.1 For every $a \in(1, \infty)$, there exists $c=c(\phi, a)>0$ such that for any $r>0$ and any open set $D \subset \bar{B}(0, r)^{c}$ we have

$$
\mathbb{P}_{x}\left(X_{\tau_{D}} \in \bar{B}(0, r)\right) \leq c r^{d} K_{D}(x, 0), \quad x \in D \cap B(0, a r)^{c} .
$$


Proof. Let $\psi \in C_{c}^{\infty}\left(\mathbb{R}^{d}\right)$ be a function such that $0 \leq \psi \leq 1$,

$$
\psi(y)= \begin{cases}0, & |y|>\frac{a+1}{2} \\ 1, & |y| \leq 1\end{cases}
$$

and $\sup _{y \in \mathbb{R}^{d}} \sum_{i, j=1}^{d}\left|\frac{\partial^{2}}{\partial y_{i} \partial y_{j}} \psi(y)\right| \leq c_{1}=c_{1}(a)$. For $r>0$ define $\psi_{r}(y):=\psi(y / r)$. Then $\psi \in$ $C_{c}^{\infty}\left(\mathbb{R}^{d}\right), 0 \leq \psi_{r} \leq 1$

$$
\psi_{r}(y)= \begin{cases}0, & |y|>\frac{a+1}{2} r \\ 1, & |y| \leq r\end{cases}
$$

and $\sup _{y \in \mathbb{R}^{d}} \sum_{i, j=1}^{d}\left|\frac{\partial^{2}}{\partial y_{i} \partial y_{j}} \psi_{r}(y)\right| \leq c_{1} r^{-2}$.

Let $x \in D \cap B(0, a r)^{c}$. Recall that $\mathcal{L}$ denotes the infinitesimal generator of $X$ and is given by (2.6). Since $\psi_{r}(x)=0$ and $D \subset \bar{B}(0, r)^{c}$, by Dynkin's formula (see, for instance, [6, (5.8)]) we have

$$
\begin{aligned}
& \mathbb{E}_{x}\left[\psi_{r}\left(X_{\tau_{D}}\right)\right]=\int_{D} G_{D}(x, z) \mathcal{L} \psi_{r}(z) d z \\
& =\int_{D \cap A(0, r,(a+2) r)} G_{D}(x, z) \mathcal{L} \psi_{r}(z) d z+\int_{D \cap \bar{B}(0,(a+2) r)^{c}} G_{D}(x, z) \mathcal{L} \psi_{r}(z) d z .
\end{aligned}
$$

For $z \in D \cap A(0, r,(a+2) r)$ we have

$$
\begin{aligned}
\left|\mathcal{L} \psi_{r}(z)\right| & =\left|\int_{\mathbb{R}^{d}}\left(\psi_{r}(z+y)-\psi_{r}(z)-\nabla \psi_{r}(z) \cdot y \mathbf{1}_{\{|y| \leq r\}}\right) j(|y|) d y\right| \\
& \leq \int_{\{|y| \leq r\}}\left|\psi_{r}(z+y)-\psi_{r}(z)-\nabla \psi_{r}(z) \cdot y\right| j(|y|) d y+2 \int_{\{r<|y|\}} j(|y|) d y \\
& \leq \frac{c_{2}}{r^{2}} \int_{\{|y| \leq r\}}|y|^{2} j(|y|) d y+2 \int_{\{r<|y|\}} j(|y|) d y \\
& \leq c_{3}\left(r^{-2} \int_{0}^{r} t \phi\left(t^{-2}\right) d t+\int_{r}^{\infty} t^{-1} \phi\left(t^{-2}\right) d t\right)
\end{aligned}
$$

where in the last line we have used (2.8). Thus by using (2.4), we get $\left|\mathcal{L} \psi_{r}(z)\right| \leq c_{4} \phi\left(r^{-2}\right)$. By Lemma 2.1 we see that $\phi\left(r^{-2}\right)$ and $\phi\left((a+2)^{-2} r^{-2}\right)$ are comparable (with a constant depending on $\phi$ and $a)$. Therefore

$$
\left|\mathcal{L} \psi_{r}(z)\right| \leq c_{4} \phi\left(|z|^{-2}\right) \leq c_{5}|z|^{d} j(|z|) \leq c_{6} r^{d} j(|z|), \quad r<|z|<(a+2) r
$$

Now assume that $z \in D \cap \bar{B}(0,(a+2) r)^{c}$. Then $\psi_{r}(z)=0$ and $\nabla \psi_{r}(z)=0$ (note that $\psi_{r}$ is zero in a neighborhood of $z$ ). Therefore

$$
\begin{aligned}
\mathcal{L} \psi_{r}(z) & =\int_{\mathbb{R}^{d}}\left(\psi_{r}(z+y)-\psi_{r}(z)-\nabla \psi_{r}(z) \cdot y \mathbf{1}_{\{|y| \leq r\}}\right) j(|y|) d y \\
& =\int_{\mathbb{R}^{d}} \psi_{r}(z+y) j(|y|) d y=\int_{|z+y| \leq \frac{a+1}{2} r} \psi_{r}(z+y) j(|y|) d y
\end{aligned}
$$


where the last equality follows from the fact that $\psi_{r}(z+y) \neq 0$ only if $|z+y| \leq \frac{a+1}{2} r$. Suppose that $|z+y| \leq \frac{a+1}{2} r$. By the triangle inequality,

$$
|y| \geq|z|-\frac{a+1}{2} r>|z|-\frac{a+1}{2} \frac{1}{a+2}|z|=\frac{a+3}{2(a+2)}|z| \geq \frac{|z|}{2} .
$$

It follows from (2.10) that $j(|y|) \leq j(|z| / 2) \leq c_{7} j(|z|)$. This implies that

$$
\mathcal{L} \psi_{r}(z) \leq c_{7} j(|z|) \int_{|z+y| \leq \frac{a+1}{2} r} d y \leq c_{8} r^{d} j(|z|) .
$$

Combining (3.2)-(3.4) we obtain

$$
\begin{aligned}
\mathbb{E}_{x}\left[\psi_{r}\left(X_{\tau_{D}}\right)\right] & =\int_{D \cap A(0, r,(a+2) r)} G_{D}(x, z) \mathcal{L} \psi_{r}(z) d z+\int_{D \cap \bar{B}(0,(a+2) r)^{c}} G_{D}(x, z) \mathcal{L} \psi_{r}(z) d z \\
& \leq c_{9} r^{d} \int_{D} G_{D}(x, z) j(|z|) d z=c_{9} r^{d} K_{D}(x, 0) .
\end{aligned}
$$

Finally, since $\mathbf{1}_{\overline{B(0, r)}} \leq \psi_{r}, \mathbb{P}_{x}\left(X_{\tau_{D}} \in \overline{B(0, r)}\right) \leq \mathbb{E}_{x}\left[\psi_{r}\left(X_{\tau_{D}}\right)\right] \leq c_{9} r^{d} K_{D}(x, 0)$.

Lemma 3.2 Let $1<p<q<\infty$. There exists $c=c(\phi, p, q)>1$ such that for all $r \geq 1 / 4$ it holds that

$$
K_{\bar{B}(0, r)^{c}}(x, z) \leq c r^{-d}\left(\phi\left(r^{-2}\right)^{-1 / 2} \phi\left((r-|z|)^{-2}\right)^{1 / 2}+1\right)
$$

for all $x \in A(0, p r, q r)$ and $z \in B(0, r)$.

Proof. We rewrite the Poisson kernel $K_{\bar{B}(0, r)^{c}}$ as follows:

$$
\begin{aligned}
& K_{\bar{B}(0, r)^{c}}(x, z)=\int_{\bar{B}(0, r)^{c}} G_{\bar{B}(0, r)^{c}}(x, y) j(|y-z|) d y \\
& =\left(\int_{A(0, r, 2 q r) \cap\{|x-z| \leq 2|x-y|\}}+\int_{A(0, r, 2 q r) \cap\{|x-z|>2|x-y|\}}+\int_{\bar{B}(0,2 q r)^{c}}\right) G_{\bar{B}(0, r)^{c}}(x, y) j(|y-z|) d y \\
& =: I_{1}+I_{2}+I_{3} .
\end{aligned}
$$

Note that, since $x \in A(0, p r, q r)$ and $z \in B(0, r)$, for $y \in A(0, r, 2 q r)$ with $|x-z| \leq 2|x-y|$, we have

$$
|x-y| \geq \frac{1}{2}(|x|-|z|) \geq \frac{1}{2}(p-1) r>(4 q)^{-1}(p-1) \delta_{\bar{B}(0, r)^{c}}(y) .
$$

We claim that when $y \in A(0, r, 2 q r)$ satisfies $|x-z| \leq 2|x-y|$,

$$
G_{\bar{B}(0, r)^{c}}(x, y) \leq c_{1} \frac{\phi\left(r^{-2}\right)^{1 / 2}}{\phi\left(\delta_{\bar{B}(0, r)^{c}}(y)^{-2}\right)^{1 / 2}} g(r) \leq c_{2} \phi\left(r^{-2}\right)^{-1 / 2} \phi\left(\delta_{\bar{B}(0, r)^{c}}(y)^{-2}\right)^{-1 / 2} r^{-d} .
$$

Since $G_{\bar{B}(0, r)^{c}}(x, y) \leq g(|x-y|)$, by (2.9) and (23.6), we only need to prove the first inequality in (3.7) for $(x, y)$ satisfying $y \in A(0, r,(p+7) r / 8)$ and $|x-z| \leq 2|x-y|$. In this case, we have

$$
|x-y| \geq \frac{1}{2}(p-1) r>4 \delta_{\bar{B}(0, r)^{c}}(y) .
$$


Let $y_{1}:=\left(8^{-1}(p-1)+1\right) r|y|^{-1} y$. Then

$$
\left|y_{1}-y\right| \leq \delta_{\bar{B}(0, r)^{c}}(y) \vee \delta_{\bar{B}(0, r)^{c}}\left(y_{1}\right) \leq \frac{1}{8}(p-1) r \leq \frac{1}{4}|x-y| .
$$

Thus by (3.8)

$$
\left|x-y_{1}\right| \geq|x-y|-\left|y_{1}-y\right| \geq \frac{3}{4}|x-y| \geq \frac{3}{8}(p-1) r .
$$

Because of (3.8), we can apply Theorem 2.9 and then use (3.9) to get that for $(x, y)$ satisfying $y \in A(0, r,(p+7) r / 8)$ and $|x-z| \leq 2|x-y|$,

$$
\begin{aligned}
& G_{\bar{B}(0, r)^{c}}(x, y) \leq c_{3} \frac{\phi\left(\delta_{\bar{B}(0, r)^{c}}\left(y_{1}\right)^{-2}\right)^{1 / 2}}{\phi\left(\delta_{\bar{B}(0, r)^{c}}(y)^{-2}\right)^{1 / 2}} G_{\bar{B}(0, r)^{c}}\left(x, y_{1}\right) \leq c_{3} \frac{\phi\left((64)(p-1)^{-2} r^{-2}\right)^{1 / 2}}{\phi\left(\delta_{\bar{B}(0, r)^{c}}(y)^{-2}\right)^{1 / 2}} g\left(\left|x-y_{1}\right|\right) \\
& \leq c_{4} \frac{\phi\left(r^{-2}\right)^{1 / 2}}{\phi\left(\delta_{\bar{B}(0, r)^{c}}(y)^{-2}\right)^{1 / 2}} g\left(\frac{3}{8}(p-1) r\right) \leq c_{5} \frac{\phi\left(r^{-2}\right)^{1 / 2}}{\phi\left(\delta_{\bar{B}(0, r)^{c}}(y)^{-2}\right)^{1 / 2}} g(r)
\end{aligned}
$$

with constants $c_{i}=c_{i}(\phi, p, q)>0, i=3,4,5$. In the last inequality we have used (3.1). Therefore using (2.9) we have proved (3.7).

Applying (3.7) to $I_{1}$ and using the fact that $\delta_{\bar{B}(0, r)^{c}}(y) \leq|y-z|$, we get

$$
I_{1} \leq c_{2} r^{-d} \phi\left(r^{-2}\right)^{-1 / 2} \int_{A(0, r, 2 q r) \cap\{|x-z|>2|x-y|\}} \phi\left(|y-z|^{-2}\right)^{-1 / 2} j(|y-z|) d y .
$$

Since $B(0, r)^{c} \subset \bar{B}(z, r-|z|)^{c}$, by (2.8) , the integral above is less than or equal to

$$
\int_{\bar{B}(z, r-|z|)^{c}} \phi\left(|y-z|^{-2}\right)^{-1 / 2} j(|y-z|) d y \leq c_{6} \int_{r-|z|}^{\infty} \frac{\phi\left(t^{-2}\right)^{1 / 2}}{t} d t \leq c_{7} \phi\left((r-|z|)^{-2}\right)^{1 / 2},
$$

where in the last inequality we used (2.5). Hence, $I_{1} \leq c_{8} \phi\left(r^{-2}\right)^{-1 / 2} \phi\left((r-|z|)^{-2}\right)^{1 / 2} r^{-d}$.

To estimate $I_{2}$ we first note that if $2|x-y| \leq|x-z|$, then $|y-z| \geq|x-z|-|y-x| \geq \frac{1}{2}|x-z|$, hence, by (2.4), $j(|y-z|) \leq c_{9} j(|x-z|)$ where $c_{9}=c_{9}(\phi)>0$. Thus,

$$
\begin{aligned}
& I_{2} \leq c_{10} j(|x-z|) \int_{B\left(x, \frac{|x-z|}{2}\right)} g(|x-y|) d y \leq c_{11} j(|x-z|) \int_{0}^{\frac{|x-z|}{2}} t^{-1} \phi\left(t^{-2}\right)^{-1} d t \\
& \leq c_{12} j(|x-z|) \phi\left(|x-z|^{-2}\right)^{-1} \leq c_{13}|x-z|^{-d} \leq c_{14} r^{-d} .
\end{aligned}
$$

In the penultimate inequality, we used (2.5).

Finally, we deal with $I_{3}$. For $|y| \geq 2 q r$ and $|z|<r$ we have that $|y-z| \geq|y|-|z|>|y|-r \geq$ $(1-1 /(2 q))|y|$, hence by (2.10), we get $j(|y-z|) \leq c_{15} j(|y|)$. Also, for $|x|<q r$ and $|y| \geq 2 q r$, we have that $|y-x| \geq|y|-|x| \geq|y| / 2$, hence $g(|x-y|) \leq c_{16} g(|y|)$ by (3.1). Therefore, by Theorem 2.3 ,

$$
I_{3} \leq c_{17} \int_{\bar{B}(0,2 q r)^{c}} \frac{1}{|y|^{d} \phi\left(|y|^{-2}\right)} \frac{\phi\left(|y|^{-2}\right)}{|y|^{d}} d y \leq c_{18} \int_{2 q r}^{\infty} t^{-d-1} d t=c_{19} r^{-d}
$$


This concludes the proof of the lemma.

It is easy to see that, by the strong Markov property, for all Greenian open sets $U$ and $D$ with $U \subset D, G_{D}(x, y)=G_{U}(x, y)+\mathbb{E}_{x}\left[G_{D}\left(X_{\tau_{U}}, y\right)\right]$ for every $(x, y) \in \mathbb{R}^{d} \times \mathbb{R}^{d}$. Thus, for all Greenian open sets $U$ and $D$ with $U \subset D$,

$$
K_{D}(x, z)=K_{U}(x, z)+\mathbb{E}_{x}\left[K_{D}\left(X_{\tau_{U}}, z\right)\right], \quad(x, z) \in U \times \bar{D}^{c}
$$

Since $X$ is a purely discontinuous rotationally invariant Lévy process, it follows from [18, Proposition 4.1] (see also [23, Theorem 1]) that if $V$ is a Lipschitz open set and $U \subset V$,

$$
\mathbb{P}_{x}\left(X_{\tau_{U}} \in \partial V\right)=0 \quad \text { and } \quad \mathbb{P}_{x}\left(X_{\tau_{U}} \in d z\right)=K_{U}(x, z) d z \quad \text { on } V^{c}
$$

Lemma 3.3 Let $1<p<q<\infty$. There exists $c=c(\phi, p, q)>1$ such that for all $r \geq 1 / 2$ and all open sets $U \subset \bar{B}(0, r)^{c}$ it holds that

$$
K_{U}(x, y) \leq c r^{-d}\left(\int_{U \cap B\left(0, \frac{1+p}{2} r\right)} K_{U}(z, y) d z+1\right), \quad \text { for all } x \in A(0, p r, q r) \cap U, y \in B(0, r) .
$$

Proof. Let $q_{1}=\frac{3+p}{4}, q_{2}=\frac{1+p}{2}$ (so that $1<q_{1}<q_{2}<p$ ) and $s \in\left[q_{1} r, q_{2} r\right]$. Then, by (3.10) and (3.11), for $x \in A(0, p r, q r) \cap U$ and $y \in B(0, r)$ it holds that

$$
\begin{aligned}
K_{U}(x, y) & =\mathbb{E}_{x}\left[K_{U}\left(X_{\tau_{U \cap \bar{B}(0, s)^{c}}}, y\right)\right]+K_{U \cap \bar{B}(0, s)^{c}}(x, y) \\
& =\int_{U \cap B(0, s)} K_{U}(z, y) K_{U \cap \bar{B}(0, s)^{c}}(x, z) d z+K_{U \cap \bar{B}(0, s)^{c}}(x, y) \\
& \leq \int_{U \cap B\left(0, q_{2} r\right)} \mathbf{1}_{\{|z|<s\}} K_{U}(z, y) K_{\bar{B}(0, s)^{c}}(x, z) d z+K_{\bar{B}(0, s)^{c}}(x, y) .
\end{aligned}
$$

Hence by Fubini's theorem,

$$
\begin{aligned}
& \left(q_{2}-q_{1}\right) r K_{U}(x, y)=\int_{q_{1} r}^{q_{2} r} K_{U}(x, y) d s \\
& \leq \int_{U \cap B\left(0, q_{2} r\right)}\left(\int_{|z|}^{q_{2} r} K_{\bar{B}(0, s)^{c}}(x, z) d s\right) K_{U}(z, y) d z+\int_{q_{1} r}^{q_{2} r} K_{\bar{B}(0, s)^{c}}(x, y) d s=: I_{1}+I_{2} .
\end{aligned}
$$

For $s \in\left[q_{1} r, q_{2} r\right]$ and $z \in U \cap B(0, s)$, we have $r<|z|<s \leq q_{2} r=\frac{1+p}{2} r<p r<|x| \leq q r \leq$ $\left(q / q_{1}\right) s$, so it follows from Lemmas 2.1 and 3.2 that

$$
\begin{aligned}
K_{\bar{B}(0, s)^{c}}(x, z) & \leq c_{1}\left(s^{-d}\left(\phi\left(s^{-2}\right)^{-1 / 2} \phi\left((s-|z|)^{-2}\right)^{1 / 2}+1\right)+s^{-d}\right) \\
& \leq c_{2}\left(r^{-d}\left(\phi\left(\left(q_{2} r\right)^{-2}\right)^{-1 / 2} \phi\left((s-|z|)^{-2}\right)^{1 / 2}+1\right)+r^{-d}\right) \\
& \leq c_{3} r^{-d}\left(\phi\left(r^{-2}\right)^{-1 / 2} \phi\left((s-|z|)^{-2}\right)^{1 / 2}+1\right)
\end{aligned}
$$


where $c_{2}=c_{2}(\phi, p, q)$ and $c_{3}=c_{3}(\phi, p, q)$. Hence,

$$
\begin{aligned}
\int_{|z|}^{q_{2} r} K_{\bar{B}(0, s)^{c}}(x, z) d s & \leq c_{3} r^{-d}\left(\phi\left(r^{-2}\right)^{-1 / 2} \int_{0}^{q_{2} r-|z|} \phi\left(t^{-2}\right)^{1 / 2} d t+q_{2} r\right) \\
& \leq c_{4} r^{-d}\left(\phi\left(r^{-2}\right)^{-1 / 2}\left(q_{2} r-|z|\right) \phi\left(\left(q_{2} r-|z|\right)^{-2}\right)^{1 / 2}+r\right)
\end{aligned}
$$

where the last inequality follows from (2.3). Since $t \mapsto t \phi\left(t^{-2}\right)^{1 / 2}$ is increasing by (2.1) and $q_{2} r-|z| \leq q_{2} r$, we have that $\left(q_{2} r-|z|\right) \phi\left(\left(q_{2} r-|z|\right)^{-2}\right)^{1 / 2} \leq q_{2} r \phi\left(\left(q_{2} r\right)^{-2}\right)^{1 / 2} \leq c_{5} r \phi\left(r^{-2}\right)^{1 / 2}$. Therefore,

$$
\int_{|z|}^{q_{2} r} K_{\bar{B}(0, s)^{c}}(x, z) d s \leq c_{6} r^{-d}\left(\phi\left(r^{-2}\right)^{-1 / 2} r \phi\left(r^{-2}\right)^{1 / 2}+r\right) \leq c_{7} r^{-d+1} .
$$

Further, for $s \in\left[q_{1} r, q_{2} r\right]$ and $y \in B(0, r)$ we have $|y|<r<q_{1} r<s$, and so $s-|y|>\left(q_{1}-1\right) r$, and we get similarly as above (but easier) that

$$
I_{2}=\int_{q_{1} r}^{q_{2} r} K_{\bar{B}(0, s)^{c}}(x, y) d s \leq c_{7} r^{-d+1}
$$

Finally,

$$
K_{U}(x, y)=\frac{1}{\left(q_{2}-q_{1}\right) r}\left(I_{1}+I_{2}\right) \leq \frac{p-1}{4} c_{8} r^{-d}\left(\int_{U \cap B\left(0, q_{2} r\right)} K_{U}(z, y) d z+1\right),
$$

proving the lemma.

Lemma 3.4 For every $a>1$ there exists $c=c(\phi, a)>1$ such that for all $r \geq 1$ and all open sets $U \subset \bar{B}(0, r)^{c}$ it holds that

$$
\frac{1}{c} K_{U}(x, 0)\left(\int_{U \cap B(0, a r)} K_{U}(y, z) d y+1\right) \leq K_{U}(x, z) \leq c K_{U}(x, 0)\left(\int_{U \cap B(0, a r)} K_{U}(y, z) d y+1\right)
$$

for all $x \in U \cap \bar{B}(0, \text { ar })^{c}$ and $z \in B(0, r)$.

Proof. Fix two constant $b_{2}$ and $b_{3}$ such that $1>b_{2}>b_{3}>1 / a$. Let $U_{1}:=\bar{B}(0, a r)^{c} \cap U$, $U_{2}:=\bar{B}\left(0, b_{2} a r\right)^{c} \cap U$ and $U_{3}:=\bar{B}\left(0, b_{3} a r\right)^{c} \cap U$. Then by (3.10), for $x \in U_{1}$ and $z \in B(0, r)$,

$$
\begin{aligned}
K_{U}(x, z) & =\mathbb{E}_{x}\left[K_{U}\left(X_{\tau_{U_{2}}}, z\right)\right]+K_{U_{2}}(x, z) \\
& =\int_{U_{3} \backslash U_{2}} K_{U}(y, z) \mathbb{P}_{x}\left(X_{\tau_{U_{2}}} \in d y\right)+\int_{U \backslash U_{3}} K_{U}(y, z) K_{U_{2}}(x, y) d y+K_{U_{2}}(x, z) \\
& =: I_{1}+I_{2}+I_{3} .
\end{aligned}
$$


We first estimate $I_{3}=K_{U_{2}}(x, z)=\int_{U_{2}} G_{U_{2}}(x, y) j(|y-z|) d y$. For $y \in U_{2}$ we have that $|y|>b_{2} a r$, hence

$$
\left(1-\frac{1}{b_{2} a}\right)|y| \leq|y|-|z| \leq|y-z| \leq|y|+|z| \leq\left(1+\frac{1}{b_{2} a}\right)|y| .
$$

Hence, by (2.10), there exists $c_{1}=c_{1}(\phi)>0$ such that $c_{1}^{-1} j(|y|) \leq j(|y-z|) \leq c_{1} j(|y|)$. Therefore,

$$
\begin{aligned}
c_{1}^{-1} K_{U_{2}}(x, 0) & =c_{1}^{-1} \int_{U_{2}} G_{U_{2}}(x, y) j(|y|) d y \leq \int_{U_{2}} G_{U_{2}}(x, y) j(|y-z|) d y \\
& =K_{U_{2}}(x, z) \leq c_{1} K_{U_{2}}(x, 0) \leq c_{1} K_{U}(x, 0) .
\end{aligned}
$$

In order to estimate $I_{2}=\int_{U \backslash U_{3}} K_{U}(y, z) K_{U_{2}}(x, y) d y$, we proceed similarly by estimating $K_{U_{2}}(x, y)=\int_{U_{2}} G_{U_{2}}(x, w) j(|w-y|) d w$ for $x \in U \cap \bar{B}(0, a r)^{c}$ and $y \in U \backslash U_{3}$. Note that since $y \notin U_{3}$ it holds that $|y|<b_{3} a r$. For $w \in U_{2}$ it holds that $|w|>b_{2} a r$. Hence, similarly as above we get that $\left(1-\frac{b_{3}}{b_{2}}\right)|w| \leq|w-y| \leq\left(1+\frac{b_{3}}{b_{2}}\right)|w|$. Thus there exists $c_{2}=c_{2}(\phi)>0$ such that $c_{2}^{-1} j(|w|) \leq j(|w-y|) \leq c_{2} j(|w|)$. In the same way as above, this implies that $c_{2}^{-1} K_{U_{2}}(x, 0) \leq K_{U_{2}}(x, y) \leq c_{2} K_{U_{2}}(x, 0) \leq c_{2} K_{U}(x, 0)$. Therefore

$$
\begin{aligned}
& c_{2}^{-1} K_{U_{2}}(x, 0) \int_{U \backslash U_{3}} K_{U}(y, z) d y \leq I_{2} \\
& \leq c_{2} K_{U_{2}}(x, 0) \int_{U \backslash U_{3}} K_{U}(y, z) d y \leq c_{2} K_{U}(x, 0) \int_{U \cap B(0, a r)} K_{U}(y, z) d y .
\end{aligned}
$$

In the case of $I_{1}$ we only need an upper estimate. It holds that

$$
I_{1}=\int_{U_{3} \backslash U_{2}} K_{U}(y, z) \mathbb{P}_{x}\left(X_{\tau_{U_{2}}} \in d y\right) \leq\left(\sup _{y \in U_{3} \backslash U_{2}} K_{U}(y, z)\right) \mathbb{P}_{x}\left(X_{\tau_{U_{2}}} \in \bar{B}\left(0, b_{2} a r\right)\right) .
$$

By Lemma 3.3 (with $p=b_{3} a$ and $q=b_{2} a$ ), there is a constant $c_{3}=c_{3}(\phi, a)>0$ such that

$$
\left(\sup _{y \in U_{3} \backslash U_{2}} K_{U}(y, z)\right) \leq c_{3} r^{-d}\left(\int_{U \backslash U_{3}} K_{U}(y, z) d y+1\right) .
$$

By Lemma 3.1 used with $D=U_{2}, b_{2} a r$ instead of $r$ and $\frac{1}{b_{2}}$ instead of $a$, there is a constant $c_{4}=c_{4}(\phi, a)>0$ such that

$$
\mathbb{P}_{x}\left(X_{\tau_{U_{2}}} \in \bar{B}\left(0, b_{2} a r\right)\right) \leq c_{4} r^{d} K_{U_{2}}(x, 0) .
$$

By applying the last two estimates to (3.18) we get

$$
I_{1} \leq c_{5} K_{U_{2}}(x, 0)\left(\int_{U \backslash U_{3}} K_{U}(y, z) d y+1\right) .
$$

Putting together (3.15), (3.17) and (3.19), we see that

$$
\begin{aligned}
K_{U}(x, z) & \leq c_{6} K_{U_{2}}(x, 0)\left(\int_{U \backslash U_{3}} K_{U}(y, z) d y+1\right) \\
& \leq c_{6} K_{U}(x, 0)\left(\int_{U \cap B(0, a r)} K_{U}(y, z) d y+1\right) .
\end{aligned}
$$


Thus, the upper bound in (3.13) holds true.

In order to prove the lower bound, we may neglect $I_{1}$. First we note that for $z \in B(0, r)$,

$$
\begin{aligned}
\int_{U \cap B(0, a r)} K_{U}(y, z) d y & =\int_{U \backslash U_{3}} K_{U}(y, z) d y+\int_{U_{3} \backslash U_{1}} K_{U}(y, z) d y \\
& \leq \int_{U \backslash U_{3}} K_{U}(y, z) d y+\left(\sup _{y \in U_{3} \backslash U_{1}} K_{U}(y, z)\right)\left|U_{3} \backslash U_{1}\right| \\
& \leq \int_{U \backslash U_{3}} K_{U}(y, z) d y+c_{3} r^{-d}\left(\int_{U \backslash U_{3}} K_{U}(y, z) d y+1\right) c_{7} r^{d} \\
& \leq c_{8}\left(\int_{U \backslash U_{3}} K_{U}(y, z) d y+1\right) .
\end{aligned}
$$

Here we used Lemma 3.3 in the second inequality. Next, by using the already proved upper bound (3.20) with $z=0$, we see that

$$
\begin{aligned}
K_{U}(x, 0) & \leq c_{6} K_{U_{2}}(x, 0)\left(\int_{U \backslash U_{3}} K_{U}(y, 0) d y+1\right) \\
& \leq c_{6} K_{U_{2}}(x, 0)\left(\int_{A(0, r, 3 r / 2)} K_{\bar{B}\left(0, \frac{r}{2}\right)^{c}}(y, 0) d y+1\right) \\
& \leq c_{6} K_{U_{2}}(x, 0)\left(\int_{A(0, r, 3 r / 2)} c_{9} r^{-d} d r+1\right) \leq c_{9} K_{U_{2}}(x, 0) .
\end{aligned}
$$

Here we have used Lemma 3.2 in the third inequality. The lower estimate now follows from (3.14), (3.16), (3.21) and (3.22).

Proof of Theorem 1.1. Let $x \in U \cap \bar{B}(0, a r)^{c}$. Then, by (3.11),

$$
\begin{aligned}
u(x) & =\int_{B(0, r)} K_{U}(x, z) u(z) d z \\
& \asymp K_{U}(x, 0) \int_{B(0, r)}\left(\int_{U \cap B(0, a r)} K_{U}(y, z) d y+1\right) u(z) d z \\
& =K_{U}(x, 0)\left(\int_{B(0, r)} u(z) d z+\int_{U \cap B(0, a r)}\left(\int_{B(0, r)} K_{U}(y, z) u(z) d z\right) d y\right) \\
& =K_{U}(x, 0)\left(\int_{B(0, r)} u(z) d z+\int_{U \cap B(0, a r)} u(y) d y\right) \\
& \asymp K_{U}(x, 0) \int_{B(0, a r)} u(z) d z,
\end{aligned}
$$

where in the second line we used Lemma 3.4 and in the first, fourth and last line we used the fact that $u$ vanishes a.e. on $\bar{B}(0, r)^{c} \backslash U$. 
Proof of Corollary 1.2, It follows from (1.4) that for $x, y \in U \cap \bar{B}(0, a r)^{c}$,

$$
\frac{u(x)}{v(x)} \leq \frac{C_{1} K_{U}(x, 0) \int_{B(0, a r)} u(z) d z}{C_{1}^{-1} K_{U}(x, 0) \int_{B(0, a r)} v(z) d z}=C_{1}^{2} \frac{\int_{B(0, a r)} u(z) d z}{\int_{B(0, a r)} v(z) d z} .
$$

Similarly,

$$
\frac{u(y)}{v(y)} \geq \frac{C_{1}^{-1} K_{U}(y, 0) \int_{B(0, a r)} u(z) d z}{C_{1} K_{U}(y, 0) \int_{B(0, a r)} v(z) d z}=C_{1}^{-2} \frac{\int_{B(0, a r)} u(z) d z}{\int_{B(0, a r)} v(z) d z} .
$$

The last two displays show that (1.5) is true for $x, y \in U \cap \bar{B}(0, a r)^{c}$ with $C_{2}=C_{1}^{4}$.

Corollary 3.5 For every $a>1$, there exists $c=c(d, \phi, a)>1$ such that

(i) for every $r \geq 1$, every open set $U \subset \bar{B}(0, r)^{c}$ and every nonnegative function $u$ on $\mathbb{R}^{d}$ which is regular harmonic in $U$ and vanishes a.e on $\bar{B}(0, r)^{c} \backslash U$,

$$
\frac{u(x)}{K_{U}(x, 0)} \leq c \frac{u(y)}{K_{U}(y, 0)}, \quad \text { for all } x, y \in U \cap \bar{B}(0, a r)^{c} ;
$$

(ii) for every $r \geq 1$ and every nonnegative function $u$ on $\mathbb{R}^{d}$ which is regular harmonic in $\bar{B}(0, r)^{c}$,

$$
\frac{u(x)}{K_{\bar{B}(0, r)^{c}}(x, 0)} \leq c \frac{u(y)}{K_{\bar{B}(0, r)^{c}}(y, 0)}, \quad \text { for all } x, y \in \bar{B}(0, a r)^{c} .
$$

Proof. The first claim is a direct consequence of Theorem 1.1 with $c=C_{1}^{2}$, while the second follows from the first and the fact that the zero boundary condition is vacuous.

Lemma 3.6 For every $a>1$, there exists $c=c(d, \phi, a)>1$ such that for all $r>0$

$$
c^{-1} r^{d} \phi\left(r^{-2}\right) G(x, 0) \leq \mathbb{P}_{x}\left(\tau_{\bar{B}(0, r)^{c}}<\infty\right) \leq c r^{d} \phi\left(r^{-2}\right) G(x, 0), \quad \text { for all } x \in \bar{B}(0, a r)^{c} .
$$

Proof. Let $x \in \bar{B}(0, a r)^{c}$. By the strong Markov property,

$$
\int_{\bar{B}(0, r)} G(x, y) d y=\mathbb{E}_{x}\left[\int_{\bar{B}(0, r)} G\left(X_{\tau_{\bar{B}(0, r)^{c}}}, y\right) d y, \tau_{\bar{B}(0, r)^{c}}<\infty\right] .
$$

Since $r \mapsto g(r)$ is decreasing, [3, Lemma 5.53] shows that there exists a constant $c=c(d)$ such that for every $r>0$ and all $z \in \bar{B}(0, r)$ we have

$$
c \int_{\bar{B}(0, r)} g(|y|) d y \leq \int_{\bar{B}(0, r)} G(z, y) d y \leq \int_{\bar{B}(0, r)} g(|y|) d y .
$$

Then it follows from (3.23) that for $x \in \bar{B}(0, a r)^{c}$,

$$
\int_{\bar{B}(0, r)} G(x, y) d y \asymp\left(\int_{B(0, r)} g(|y|) d y\right) \mathbb{P}_{x}\left(\tau_{\bar{B}(0, r)^{c}}<\infty\right),
$$


with a constant depending on $d$ only. By the uniform Harnack inequality (Theorem 2.7), there exists $c_{2}>1$ such that $c_{2}^{-1} G(x, 0) \leq G(x, y) \leq c_{2} G(x, 0)$ for every $x \in \bar{B}(0, a r)^{c}$ and $y \in B(0, r)$. Hence

$$
r^{d} G(x, 0) \asymp\left(\int_{B(0, r)} g(|y|) d y\right) \mathbb{P}_{x}\left(\tau_{\bar{B}(0, r)^{c}}<\infty\right), \quad x \in \bar{B}(0, a r)^{c},
$$

with a constant depending on $d$ and $a$. It follows from (2.9) and (2.5) that

$$
\int_{B(0, r)} g(|y|) d y \asymp \frac{1}{\phi\left(r^{-2}\right)},
$$

with a constant depending on $d$ and $a$. Combining (3.24)-(3.25) we have proved the lemma

Corollary 3.7 For every $a>1$, there exists $c=c(\phi, d, a)>1$ such that for all $r \geq 1$ it holds that

$$
c^{-1} \phi\left(r^{-2}\right) G(x, 0) \leq K_{\bar{B}(0, r)^{c}}(x, 0) \leq c \phi\left(r^{-2}\right) G(x, 0), \quad x \in \bar{B}(0, a r)^{c},
$$

and consequently

$$
\lim _{|x| \rightarrow \infty} K_{\bar{B}(0, r)^{c}}(x, 0)=0 .
$$

Proof. Note that $\mathbb{P}_{x}\left(\tau_{\bar{B}(0, r)^{c}}<\infty\right)=\int_{B(0, r)} K_{\bar{B}(0, r)^{c}}(x, z) d z$. Further, for $z \in B(0, r)$ and $y \in \bar{B}(0, r)^{c}$ we have that $|y-z| \leq 2|y|$ and hence $j(|y-z|) \geq c_{1} j(|y|)$ by (2.10). Therefore,

$$
K_{\bar{B}(0, r)^{c}}(x, z) \geq c_{1} \int_{\bar{B}(0, r)^{c}} G_{\bar{B}(0, r)^{c}}(x, y) j(|y|) d y=c_{1} K_{\bar{B}(0, r)^{c}}(x, 0) .
$$

Using (3.11) it follows that $\mathbb{P}_{x}\left(\tau_{\bar{B}(0, r)^{c}}<\infty\right) \geq c_{1} \int_{B(0, r)} K_{\bar{B}(0, r)^{c}}(x, 0) d z=c_{2} r^{d} K_{\bar{B}(0, r)^{c}}(x, 0)$. On the other hand, from Lemma 3.1 with $D=\bar{B}(0, r)^{c}$, we see that $\mathbb{P}_{x}\left(\tau_{\bar{B}(0, r)^{c}}<\infty\right) \leq c_{2} r^{d} K_{\bar{B}(0, r)^{c}}(x, 0)$. Thus

$$
\mathbb{P}_{x}\left(\tau_{\bar{B}(0, r)^{c}}<\infty\right) \asymp r^{d} K_{\bar{B}(0, r)^{c}}(x, 0), \quad x \in \bar{B}(0, a r)^{c} .
$$

Comparing with the result in Lemma 3.6 gives (3.26). The last statement follows from $\lim _{|x| \rightarrow \infty} G(x, 0)=$ 0 .

Corollary 3.8 Let $r \geq 1$ and $U \subset \bar{B}(0, r)^{c}$. If $u$ is a non-negative function on $\mathbb{R}^{d}$ which is regular harmonic in $U$ and vanishes a.e. on $\bar{B}(0, r)^{c} \backslash U$, then

$$
\lim _{|x| \rightarrow \infty} u(x)=0 .
$$

Proof. Note that $K_{U}(x, 0) \leq K_{\bar{B}(0, r)^{c}}(x, 0)$. It follows from Corollary 3.7 that

$$
\lim _{|x| \rightarrow \infty} K_{\bar{B}(0, r)^{c}}(x, 0)=0 .
$$

Then the claim follows from Theorem 1.1 , 
Remark 3.9 (i) Corollary 3.8 is not true if regular harmonic is replaced by harmonic. Indeed, let $V$ denote the renewal function of the ladder height process of the one-dimensional subordinate Brownian motion $W^{d}\left(S_{t}\right)$. Then the function $w(x)=w\left(\widetilde{x}, x_{d}\right):=V\left(\left(x_{d}\right)^{+}\right)$is harmonic in the upper half-space $\mathbb{H} \subset B((\widetilde{0},-1), 1)^{c}$ (see [11]), vanishes on $B((\widetilde{0},-1), 1)^{c} \backslash \mathbb{H}$, but clearly $\lim _{x_{d} \rightarrow \infty} w(x)=$ $\infty$.

(ii) When $d=1 \leq \alpha$, Corollary 3.8 is not true even for the symmetric $\alpha$-stable process because the Green function of the complement of any bounded interval does not vanish at infinity, which can be seen using the Kelvin transform.

\section{The infinite part of the Martin boundary}

In this section we will consider a large class of unbounded open sets $D$ and identify the infinite part of the Martin boundary of $D$ without assuming that the finite part of the Martin boundary of $D$ coincides with the Euclidean boundary.

We first recall the definition of $\kappa$-fatness at infinity from the introduction: Let $\kappa \in(0,1 / 2]$. An open set $D$ in $\mathbb{R}^{d}$ is $\kappa$-fat at infinity if there exists $R>0$ such that for every $r \in[R, \infty)$ there exists $A_{r} \in \mathbb{R}^{d}$ such that $B\left(A_{r}, \kappa r\right) \subset D \cap \bar{B}(0, r)^{c}$ and $\left|A_{r}\right|<\kappa^{-1} r$.

The origin does not play any special role in this definition: Suppose that $D$ is $\kappa$-fat at infinity with characteristics $(R, \kappa)$. For every $Q \in \mathbb{R}^{d}$, define $R_{Q}:=R \vee|Q|$. For all $r \geq R_{Q}$, with $\widehat{A}_{r}:=A_{2 r}$ and $\widehat{\kappa}:=\kappa / 3$, we have

$$
B\left(\widehat{A}_{r}, \widehat{\kappa} r\right) \subset B\left(A_{2 r}, 2 \kappa r\right) \subset D \cap \bar{B}(0,2 r)^{c} \subset D \cap \bar{B}(0, r+|Q|)^{c} \subset D \cap \bar{B}(Q, r)^{c}
$$

and $\left|\widehat{A}_{r}-Q\right| \leq\left|A_{2 r}\right|+|Q| \leq(\kappa / 2)^{-1} r+r<(\widehat{\kappa})^{-1} r$.

Remark 4.1 (i) Note that it follows from the definition that any open set which is $\kappa$-fat at infinity is necessarily unbounded.

(ii) Since $B\left(A_{r}, \kappa r\right) \subset \bar{B}(0, r)^{c}$ we have that $\left|A_{r}\right|-\kappa r>r$ implying $(\kappa+1) r<\left|A_{r}\right|<\kappa^{-1} r$.

(iii) We further note that $B\left(A_{r},(\kappa / 2) r\right) \cap B\left(A_{(\kappa / 2)^{-1} r}, r\right)=\emptyset$. Indeed, for any point $x$ in the intersection we would have that $|x| \leq\left|A_{r}\right|+(\kappa / 2) r<\left(\kappa^{-1}+\kappa / 2\right) r$, and at the same time $|x| \geq$ $\left|A_{(\kappa / 2)^{-1} r}\right|-r>(\kappa+1)(\kappa / 2)^{-1} r-\kappa r=\left(2 \kappa^{-1}+2-\kappa\right) r$. But this is impossible.

In this section we first identify the infinite part of the Martin boundary of an open set $D \subset \mathbb{R}^{d}$ which is $\kappa$-fat at infinity with characteristics $(R, \kappa)$. Without loss of generality, we assume that $R>1$.

Recall that, throughout the paper we assume that $(\mathbf{H 1})$ and (H2) are true and $d>2\left(\delta_{2} \vee \delta_{4}\right)$.

Lemma 4.2 Let $D \subset \mathbb{R}^{d}$ be an open set which is $\kappa$-fat at infinity with characteristics $(R, \kappa)$. There exist $c=c(d, \phi, \kappa)>0$ and $\gamma=\gamma(d, \phi, \kappa) \in(0, d)$ such that for every $r \geq R$ and any non-negative function $h$ in $\mathbb{R}^{d}$ which is harmonic in $D \cap \bar{B}(0, r)^{c}$ it holds that

$$
h\left(A_{r}\right) \leq c(\kappa / 2)^{-(d-\gamma) k} h\left(A_{(\kappa / 2)^{-k} r}\right), \quad k=0,1,2, \ldots .
$$


Proof. Fix $r \geq R$. For $n=0,1,2, \ldots$, let $\eta_{n}=(\kappa / 2)^{-n} r, A_{n}=A_{\eta_{n}}$ and $B_{n}=B\left(A_{n}, \eta_{n-1}\right)$ (where $\left.\eta_{-1}=(\kappa / 2) r\right)$. Note that the balls $B_{n}$ are pairwise disjoint (cf. Remark 4.1 (iii)). By harmonicity of $h$, for every $n=0,1,2 \ldots$,

$$
h\left(A_{n}\right)=\mathbb{E}_{A_{n}}\left[h\left(X_{\tau_{B_{n}}}\right)\right] \geq \sum_{l=0}^{n-1} \mathbb{E}_{A_{n}}\left[h\left(X_{\tau_{B_{n}}}\right): X_{\tau_{B_{n}}} \in B_{l}\right]=\sum_{l=0}^{n-1} \int_{B_{l}} K_{B_{n}}\left(A_{n}, z\right) h(z) d z .
$$

By the uniform Harnack inequality, Theorem [2.7, there exists $c_{1}=c_{1}(d, \kappa, \phi)>0$ such that for every $l=0,1,2, \ldots, h(z) \geq c_{1} h\left(A_{l}\right)$ for all $z \in B_{l}$. Hence

$$
\int_{B_{l}} K_{B_{n}}\left(A_{n}, z\right) h(z) d z \geq c_{1} h\left(A_{l}\right) \int_{B_{l}} K_{B_{n}}\left(A_{n}, z\right) d z, \quad 0 \leq l \leq n-1 .
$$

By (2.15) we have

$$
\int_{B_{l}} K_{B_{n}}\left(A_{n}, z\right) d z \geq c_{2} \phi\left(\eta_{n}^{-2}\right)^{-1} \int_{B_{l}} j\left(\left|2\left(A_{n}-z\right)\right|\right) d z, \quad 0 \leq l \leq n-1 .
$$

For $z \in B_{l}, l=0,1, \cdots, n-1$, it holds that $|z| \leq \kappa^{-1}(\kappa / 2)^{-l} r+(\kappa / 2)^{-(l-1)} r=(\kappa / 2)^{-l} r\left(\kappa^{-1}+\kappa / 2\right)$. Since $\left|A_{n}\right| \leq \kappa^{-1} \eta_{n}$, we have that $\left|A_{n}-z\right| \leq\left|A_{n}\right|+|z| \leq 2 \kappa^{-1} \eta_{n}$. Together with Theorem 2.3 and Lemma 2.1, this implies that $j\left(\left|2\left(A_{n}-z\right)\right|\right) \geq c_{3} j\left(\left|\eta_{n}\right|\right)$ for every $z \in B_{l}$ and $0 \leq l \leq n-1$. Therefore,

$$
\int_{B_{l}} K_{B_{n}}\left(A_{n}, z\right) d z \geq c_{4} j\left(\left|\eta_{n}\right|\right) \phi\left(\eta_{n}^{-2}\right)^{-1}\left|B_{l}\right| \geq c_{5} \eta_{n}^{-d} \eta_{l}^{d}=c_{5} \frac{\eta_{l}^{d}}{\eta_{n}^{d}}, \quad 0 \leq l \leq n-1 .
$$

Hence,

$$
\eta_{n}^{d} h\left(A_{n}\right) \geq c_{5} \sum_{l=0}^{n-1} \eta_{l}^{d} h\left(A_{l}\right), \quad \text { for all } n=1,2, \ldots
$$

Let $a_{n}:=\eta_{n}^{d} h\left(A_{n}\right)$ so that $a_{n} \geq c_{5} \sum_{l=0}^{n-1} a_{l}$. Using the identity $1+c_{5}+c_{5} \sum_{l=1}^{n-2}\left(1+c_{5}\right)^{l}=\left(1+c_{5}\right)^{n-1}$ for $n \geq 3$, by induction it follows that $a_{n} \geq c_{5}\left(1+c_{5}\right)^{n-1} a_{0}$. Let $\gamma:=\log \left(1+c_{5}\right) / \log (2 / \kappa)$ so that $\left(1+c_{5}\right)^{n}=(2 / \kappa)^{\gamma n}$. Note that $c_{5}$ can be chosen arbitrarily close to zero (but positive), so that $\gamma<d$. Thus, $a_{0} \leq\left(1+c_{5}\right) c_{5}^{-1}(\kappa / 2)^{\gamma n} a_{n}$, or $\eta_{0}^{d} h\left(A_{0}\right) \leq\left(1+c_{5}\right) c_{5}^{-1}(\kappa / 2)^{\gamma n} \eta_{n}^{d} h\left(A_{n}\right)$. Hence, $h\left(A_{r}\right) \leq\left(1+c_{5}\right) c_{5}^{-1}(\kappa / 2)^{\gamma n}(\kappa / 2)^{-d n} h\left(A_{(\kappa / 2)^{-n} r}\right)$.

Lemma 4.3 Let $D \subset \mathbb{R}^{d}$ be an open set which is $\kappa$-fat at infinity with characteristics $(R, \kappa)$. There exists $c=c(d, \phi, \kappa)>0$ such that for every $r \geq R$ and every non-negative function $h$ on $\mathbb{R}^{d}$ which is regular harmonic in $D \cap \bar{B}(0,(\kappa / 2+1) r)^{c}$, it holds that

$$
h\left(A_{r}\right) \geq c r^{-d} \int_{B(0, r)} h(z) d z .
$$


Proof. Since $h$ is regular harmonic in $D \cap \bar{B}(0,(\kappa / 2+1) r)^{c}$ and $B\left(A, \frac{\kappa r}{2}\right) \subset D \cap \bar{B}(0,(\kappa / 2+1) r)^{c}$, we have

$$
h\left(A_{r}\right)=\mathbb{E}_{A_{r}}\left[h\left(X_{\left.\tau_{B\left(A_{r}, \frac{\kappa r}{2}\right)}\right)}\right) \geq \int_{B(0, r)} K_{B\left(A_{r}, \frac{\kappa r}{2}\right)}\left(A_{r}, z\right) h(z) d z .\right.
$$

By (2.15) we have

$$
K_{B\left(A_{r}, \frac{\kappa r}{2}\right)}\left(A_{r}, z\right) \geq c_{1} j\left(\left|2\left(A_{r}-z\right)\right|\right) \phi\left(\left(\frac{\kappa r}{2}\right)^{-2}\right)^{-1}, \quad z \in B(0, r) .
$$

Since for $z \in B(0, r)$ we have that $\left|A_{r}-z\right|<\left(\kappa^{-1}+1\right) r$, by (2.10) we have $j\left(\left|2\left(A_{r}-z\right)\right|\right) \geq c_{2} j(r)$ for some constant $c_{2}=c_{2}(\phi, \kappa)>0$. Hence, combining (4.2)-(4.3) and applying Lemma 2.1, we get

$$
h\left(A_{r}\right) \geq \int_{B(0, r)} j(r) \phi\left(r^{-2}\right)^{-1} h(z) d z \geq c_{3} r^{-d} \int_{B(0, r)} h(z) d z
$$

which finishes the proof.

Corollary 4.4 Let $D \subset \mathbb{R}^{d}$ be an open set which is $\kappa$-fat at infinity with characteristics $(R, \kappa)$. There exists $c=c(d, \phi, \kappa)>0$ such that for every $r \geq R$ with $D \cap B(0, r) \neq \emptyset$ and every $w \in$ $D \cap B(0, r)$ it holds that

$$
G_{D}\left(A_{r}, w\right) \geq c r^{-d} \int_{B(0, r)} G_{D}(z, w) d z
$$

Proof. Let $h(\cdot):=G_{D}(\cdot, w)$. Then $h$ is regular harmonic in $D \cap \bar{B}(0,(\kappa / 2+1) r)^{c}$ so the claim follows from Lemma 4.3 .

Lemma 4.5 Let $D \subset \mathbb{R}^{d}$ be an open set which is $\kappa$-fat at infinity with characteristics $(R, \kappa)$. For $r>0$ and $n=0,1,2, \ldots$, let $B_{n}(r)=B\left(0,(\kappa / 2)^{-n} r\right)$. There exist $c_{1}=c_{1}(d, \phi, \kappa)>0$ and $c_{2}=c_{2}(d, \phi, \kappa) \in(0,1)$ such that for any $r \geq R$ and any non-negative function $h$ which is regular harmonic in $D \cap \bar{B}(0, r)^{c}$ and vanishes in $D^{c} \cap \bar{B}(0, r)^{c}$ we have

$$
\mathbb{E}_{x}\left[h\left(X_{\tau_{D \cap \overline{B n}^{(r)}}^{c}}\right): X_{\tau_{D \cap \overline{B n}^{(r)}}^{c}} \in B(0, r)\right] \leq c_{1} c_{2}^{n} h(x), \quad x \in D \cap{\overline{B_{n}(r)}}^{c}, n=0,1,2, \ldots
$$

Proof. We fix $r \geq R$. For $n=0,1,2, \ldots$, let $B_{n}=B_{n}(r), \bar{B}_{n}=\overline{B_{n}(r)}$ and $\eta_{n}=(\kappa / 2)^{-n} r$, and define

$$
h_{n}(x):=\mathbb{E}_{x}\left[h\left(X_{\tau_{D \cap \bar{B}_{n}^{c}}}\right): X_{\tau_{D \cap \bar{B}_{n}^{c}}} \in B_{0}\right], \quad x \in D \cap \bar{B}_{n}^{c} .
$$

Then for $x \in D \cap \bar{B}_{n+1}^{c}$ we have

$$
h_{n+1}(x)=\mathbb{E}_{x}\left[h\left(X_{\tau_{D \cap \bar{B}_{n}^{c}}}\right): \tau_{D \cap \bar{B}_{n+1}^{c}}=\tau_{D \cap \bar{B}_{n}^{c}}, X_{\tau_{D \cap \bar{B}_{n}^{c}}} \in B_{0}\right] \leq h_{n}(x) .
$$


Let $A_{n}=A_{\eta_{n}}$. Then

$h_{n}\left(A_{n}\right)=\mathbb{E}_{A_{n}}\left[h\left(X_{\tau_{D \cap \bar{B}_{n}^{c}}}\right): X_{\tau_{D \cap \bar{B}_{n}^{c}}} \in B_{0}\right] \leq \mathbb{E}_{A_{n}}\left[h\left(X_{{\overline{\bar{B}_{n}^{c}}}^{c}}\right): X_{\tau_{\bar{B}_{n}^{c}}} \in B_{0}\right]=\int_{B_{0}} K_{\bar{B}_{n}^{c}}\left(A_{n}, z\right) h(z) d z$.

By Lemma 3.2, there exists $c_{1}=c_{1}(\phi, \kappa)>0$ such that

$$
K_{\bar{B}_{n}^{c}}\left(A_{n}, z\right) \leq c_{1}\left(\left|A_{n}-z\right|^{-d}\left(\phi\left(\eta_{n}^{-2}\right)^{-1 / 2} \phi\left(\left(\eta_{n}-|z|\right)^{-2}\right)^{1 / 2}\right)+\eta_{n}^{-d}\right) .
$$

For $z \in B_{0}$ and $n \geq 1$ we have that $\left|A_{n}-z\right| \asymp \eta_{n}$ and $\eta_{n}-|z| \asymp \eta_{n}$, thus

$$
K_{\bar{B}_{n}^{c}}\left(A_{n}, z\right) \leq c_{2} \eta_{n}^{-d}=c_{2}(\kappa / 2)^{n d} r^{-d}, \quad z \in B_{0}, n=1,2,3 \ldots
$$

Therefore, by Lemma 4.3 in the second inequality below and Lemma 4.2 in the third, we get that for $n=1,2,3 \ldots$,

$$
h_{n}\left(A_{n}\right) \leq c_{2}(\kappa / 2)^{n d} r^{-d} \int_{B_{0}} h(z) d z \leq c_{3}(\kappa / 2)^{n d} h\left(A_{0}\right) \leq c_{4}(\kappa / 2)^{\gamma n} h\left(A_{n}\right),
$$

where $\gamma \in(0, d)$ is the constant from Lemma 4.2. Now note that both $h_{n-1}$ and $h$ are regular harmonic in $D \cap \bar{B}_{n-1}^{c}$ and vanish on $B_{n-1}^{c} \cap D^{c}=B_{n-1}^{c} \backslash D \cap \bar{B}_{n-1}^{c}$. Hence,

$$
\frac{h_{n}(x)}{h(x)} \leq \frac{h_{n-1}(x)}{h(x)} \leq c_{5} \frac{h_{n-1}\left(A_{n-1}\right)}{h\left(A_{n-1}\right)} \leq c_{5} C_{2}(\kappa / 2)^{\gamma n}, \quad x \in D \cap \bar{B}_{n}^{c} \quad n=2,3,4 \ldots
$$

where the second inequality follows from Corollary 1.2. The cases $n=0$ and $n=1$ are clear by the harmonicity of $h$.

Corollary 4.6 Let $D \subset \mathbb{R}^{d}$ be an open set which is $\kappa$-fat at infinity with characteristics $(R, \kappa)$. For $r>0$ and $n=0,1,2, \ldots$, let $B_{n}(r)=B\left(0,(\kappa / 2)^{-n} r\right)$. There exist $c_{1}=c_{1}(d, \phi, \kappa)>0$ and $c_{2}=c_{2}(d, \phi, \kappa) \in(0,1)$ such that for any $r \geq R$ with $D \cap B(0, r) \neq \emptyset$, any $w \in D \cap B(0, r)$ and $n \geq 0$, we have

$$
\mathbb{E}_{x}\left[G_{D}\left(X_{\tau_{D \cap \overline{B n}^{(r)}}}, w\right): X_{\tau_{D \cap \overline{B n}^{(r)}}^{c}} \in B(0, r)\right] \leq c_{1} c_{2}^{n} G_{D}(x, w), \quad x \in D \cap{\overline{B_{n}(r)}}^{c} .
$$

The following lemma is an analog of [2, Lemma 16] for infinity. The proof is essentially the same - instead of using the balls that shrink to a finite boundary point, we use concentric balls with larger and larger radius (so they "shrink at infinity"). Lemmas 13 and 14 from [2] are replaced by our Corollary 1.2 and Lemma 4.5 respectively. Below we only indicate essential changes in the proof and refer the reader to the proof of [2, Lemma 16].

Lemma 4.7 Let $D \subset \mathbb{R}^{d}$ be an open set which is $\kappa$-fat at infinity with characteristics $(R, \kappa)$. There exist $c=c(d, \phi, \kappa)>0$ and $\nu=\nu(d, \phi, \kappa)>0$ such that for any $r \geq R$ and all non-negative 
functions $u$ and $v$ on $\mathbb{R}^{d}$ which are regular harmonic in $D \cap \bar{B}(0, r / 2)^{c}$, vanish in $D^{c} \cap \bar{B}(0, r / 2)^{c}$ and satisfy $u\left(A_{r}\right)=v\left(A_{r}\right)$, there exists the limit

$$
I(u, v)=\lim _{|x| \rightarrow \infty, x \in D} \frac{u(x)}{v(x)},
$$

and we have

$$
\left|\frac{u(x)}{v(x)}-I(u, v)\right| \leq c\left(\frac{|x|}{r}\right)^{-\nu}, \quad x \in D \cap \bar{B}(0, r)^{c} .
$$

Proof. Let $r \geq R$ be fixed. Without loss of generality assume that $u\left(A_{r}\right)=v\left(A_{r}\right)=1$. Let $n_{0}(d, \phi) \in \mathbb{N}$ to be chosen later, and let $a=(\kappa / 2)^{-n_{0}}$. For $n=0,1,2, \ldots$, define

$$
r_{n}=a^{n} r, \quad \bar{B}_{n}^{c}=\bar{B}\left(0, r_{n}\right)^{c}, \quad \bar{D}_{n}^{c}=D \cap \bar{B}_{n}^{c}, \quad \Pi_{n}=\bar{D}_{n}^{c} \backslash \bar{D}_{n+1}^{c}, \quad \Pi_{-1}=B(0, r) .
$$

For $l=-1,0,1, \ldots, n-1$ let

$$
\begin{array}{lll}
u_{n}^{l}(x):=\mathbb{E}_{x}\left[u\left(X_{\tau_{\bar{D}_{n}^{c}}}\right): X_{\tau_{\bar{D}_{n}^{c}}} \in \Pi_{l}\right], & x \in \mathbb{R}^{d}, \\
v_{n}^{l}(x):=\mathbb{E}_{x}\left[v\left(X_{\tau_{\bar{D}_{n}^{c}}}\right): X_{\tau_{\bar{D}_{n}^{c}}} \in \Pi_{l}\right], & x \in \mathbb{R}^{d} .
\end{array}
$$

Note that since $\Pi_{l} \subset B\left(0, r_{l+1}\right)$, it holds that

$$
u_{n}^{l}(x) \leq \mathbb{E}_{x}\left[u\left(X_{\tau_{\bar{D}_{n}^{c}}}\right): X_{\tau_{\bar{D}_{n}^{c}}} \in B\left(0, r_{l+1}\right)\right] .
$$

Denote the constants $c_{1}$ and $c_{2}$ in Lemma 4.5 by $\widetilde{C}$ and $\xi$ respectively. Apply Lemma 4.5 with $\tilde{r}=r_{l+1}$. Then $r_{n}=(\kappa / 2)^{-n_{0} n} r=(\kappa / 2)^{-n_{0}(n-l-1)} \tilde{r}$, hence for $n=0,1,2, \ldots$ and $x \in \bar{D}_{n}^{c}$,

$$
u_{n}^{l}(x) \leq \widetilde{C}\left(\xi^{n_{0}}\right)^{n-l-1} u(x), \quad l=-1,0,1, \ldots, n-2 .
$$

Choose $n_{0}$ large enough so that $\left(1-\xi^{n_{0}}\right)^{-1} \leq 2$. Then since $\sum_{l=-1}^{n-2}\left(\xi^{n_{0}}\right)^{n-l-1}=\xi^{n_{0}} \sum_{n=0}^{n-1}\left(\xi^{n_{0}}\right)^{n} \leq$ $\xi^{n_{0}}\left(1-\xi^{n_{0}}\right)^{-1} \leq 2 \xi^{n_{0}}$, we have that for $n=1,2, \ldots$ and $x \in \bar{D}_{n}^{c}$,

$$
\sum_{l=-1}^{n-2} u_{n}^{l}(x) \leq 2 \widetilde{C} \xi^{n_{0}} u(x) .
$$

For any $\epsilon \in(0,1)$, we can redefine $n_{0}(\epsilon, d, \phi)$ so that for $n=1,2, \ldots, l=-1,0,1, \ldots, n-2$,

$$
u_{n}^{l}(x) \leq \epsilon^{n-1-l} u_{n}^{n-1}(x), \quad x \in \bar{D}_{n}^{c} .
$$

By symmetry we can also achieve that for $n=1,2, \ldots, l=-1,0,1, \ldots, n-2$,

$$
v_{n}^{l}(x) \leq \epsilon^{n-1-l} v_{n}^{n-1}(x), \quad x \in \bar{D}_{n}^{c} .
$$

Now we claim that there exist constants $c_{1}=c_{1}(d, \phi, \kappa)>0$ and $\zeta=\zeta(d, \phi, \kappa) \in(0,1)$ such that for all $l=0,1, \ldots$,

$$
\sup _{x \in \bar{D}_{l}^{c}} \frac{u(x)}{v(x)} \leq\left(1+c_{1} \zeta^{l}\right) \inf _{x \in \bar{D}_{l}^{c}} \frac{u(x)}{v(x)} .
$$

From now on the proof is essentially the same as the proof of [2, Lemma 16], hence we omit it. 
Remark 4.8 (i) Assume that $u$ and $v$ are nonnegative functions on $\mathbb{R}^{d}$ which are regular harmonic in $D \cap \bar{B}(0, r / 2)^{c}$ and vanish in $D^{c} \cap \bar{B}(0, r / 2)^{c}$. Define $\widetilde{u}_{r}$ and $\widetilde{v}_{r}$ by

$$
\widetilde{u}_{r}(x):=\frac{u(x)}{u\left(A_{r}\right)}, \quad \widetilde{v}_{r}(x):=\frac{v(x)}{v\left(A_{r}\right)} .
$$

Then $\widetilde{u}_{r}$ and $\widetilde{v}_{r}$ satisfy assumptions of Lemma 4.7, in particular $\widetilde{u}_{r}\left(A_{r}\right)=\widetilde{v}_{r}\left(A_{r}\right)$. Hence, there exists the limit

$$
I\left(\widetilde{u}_{r}, \widetilde{v}_{r}\right)=\lim _{|x| \rightarrow \infty, x \in D} \frac{\widetilde{u}_{r}(x)}{\widetilde{v}_{r}(x)} .
$$

Therefore we can conclude that there exists the limit

$$
I\left(u, v, A_{r}\right)=\lim _{|x| \rightarrow \infty, x \in D} \frac{u(x)}{v(x)}=\frac{u\left(A_{r}\right)}{v\left(A_{r}\right)} I\left(\widetilde{u}_{r}, \widetilde{v}_{r}\right) .
$$

Suppose that $\rho \geq R$ is another radius such that $u$ and $v$ are regular harmonic in $D \cap \bar{B}(0, \rho / 2)^{c}$ and vanish in $D^{c} \cap \bar{B}(0, \rho / 2)^{c}$. Then the same argument using $A_{\rho}$ instead of $A_{r}$ would give that there exists the limit

$$
I\left(u, v, A_{\rho}\right)=\lim _{|x| \rightarrow \infty, x \in D} \frac{u(x)}{v(x)}=\frac{u\left(A_{\rho}\right)}{v\left(A_{\rho}\right)} I\left(\widetilde{u}_{\rho}, \widetilde{v}_{\rho}\right) .
$$

This shows that the limit is independent of the point $A_{r}$.

(ii) It easily follows from (4.6) that there exist $c=c(d, \phi, \kappa)>0$ and $\nu=\nu(d, \phi, \kappa)>0$ such that for any $r \geq R$,

$$
\left|\frac{u(x)}{v(x)}-\frac{u(y)}{v(y)}\right| \leq c\left|\frac{x-y}{r}\right|^{-\nu} \quad \forall x, y \in D \cap \bar{B}(0, r)^{c}
$$

for all non-negative functions $u$ and $v$ on $\mathbb{R}^{d}$ which are regular harmonic in $D \cap \bar{B}(0, r / 2)^{c}$, vanish in $D^{c} \cap \bar{B}(0, r / 2)^{c}$ and satisfy $u\left(A_{r}\right)=v\left(A_{r}\right)$.

From now on $D$ will be an open set which is $\kappa$-fat at infinity with characteristics $(R, \kappa)$. Fix $x_{0} \in D \cap \bar{B}(0, R)^{c}$ and recall that

$$
M_{D}(x, y)=\frac{G_{D}(x, y)}{G_{D}\left(x_{0}, y\right)}, \quad x, y \in D \cap \bar{B}(0, R)^{c} .
$$

For $r>(2|x| \vee R)$, both functions $y \mapsto G_{D}(x, y)$ and $y \mapsto G_{D}\left(x_{0}, y\right)$ are regular harmonic in $D \cap \bar{B}(0, r / 2)^{c}$ and vanish on $D^{c} \cap \bar{B}(0, r / 2)^{c}$. Hence, as an immediate consequence of Lemma 4.7 and Remark 4.8 (i) we get the following theorem.

Theorem 4.9 For each $x \in D$ there exists the limit

$$
M_{D}(x, \infty):=\lim _{y \in D,|y| \rightarrow \infty} M_{D}(x, y)
$$


Recall that $X^{D}$ is the process $X$ killed upon exiting $D$. As the process $X^{D}$ satisfies Hypothesis (B) in [16], $D$ has a Martin boundary $\partial_{M} D$ with respect to $X$ satisfying the following properties:

(M1) $D \cup \partial_{M} D$ is a compact metric space (with the metric denoted by $d$ );

(M2) $D$ is open and dense in $D \cup \partial_{M} D$, and its relative topology coincides with its original topology;

(M3) $M_{D}(x, \cdot)$ can be uniquely extended to $\partial_{M} D$ in such a way that

(a) $M_{D}(x, y)$ converges to $M_{D}(x, w)$ as $y \rightarrow w \in \partial_{M} D$ in the Martin topology,

(b) for each $w \in D \cup \partial_{M} D$ the function $x \rightarrow M_{D}(x, w)$ is excessive with respect to $X^{D}$,

(c) the function $(x, w) \rightarrow M_{D}(x, w)$ is jointly continuous on $D \times\left(D \cup \partial_{M} D\right)$ in the Martin topology and

(d) $M_{D}\left(\cdot, w_{1}\right) \neq M_{D}\left(\cdot, w_{2}\right)$ if $w_{1} \neq w_{2}$ and $w_{1}, w_{2} \in \partial_{M} D$.

In the remainder of the paper whenever we speak of a bounded or an unbounded sequence of points we always mean in the Euclidean metric (and not in the Martin metric $d$ ).

Definition 4.10 A point $w \in \partial_{M} D$ is called a finite Martin boundary point if there exists a bounded sequence $\left(y_{n}\right)_{n \geq 1}, y_{n} \in D$, converging to $w$ in the Martin topology. A point $w \in \partial_{M} D$ is called an infinite Martin boundary point if every sequence $\left(y_{n}\right)_{n \geq 1}, y_{n} \in D$, converging to $w$ in the Martin topology is unbounded. The set of finite Martin boundary points is denoted by $\partial_{M}^{f} D$, and the set of infinite Martin boundary points by $\partial_{M}^{\infty} D$.

Remark 4.11 Suppose that $w \in \partial_{M}^{f} D$ and let $\left(y_{n}\right)_{n \geq 1} \subset D$ be a bounded sequence converging to $w$ in the Martin topology. Then $\left(y_{n}\right)_{n \geq 1}$ has a subsequence $\left(y_{n_{k}}\right)_{k \geq 1}$ converging to a point $y$ in the Euclidean topology. It cannot happen that $y \in D$, because in this case we would have that $\lim _{y_{n_{k}} \rightarrow y} M_{D}\left(x, y_{n_{k}}\right)=M_{D}(x, y)$ implying by $(\mathrm{M} 3)(\mathrm{d})$ that $y=w$. Therefore, $y \in \partial D-$ the Euclidean boundary of $D$. In particular, this shows that for every $\epsilon>0$, the sequence $\left(y_{n}\right)_{n \geq 1}$ (converging to $w \in \partial_{M}^{f} D$ in the Martin topology) can be chosen so that $\delta_{D}\left(y_{n}\right)<\epsilon$ for all $n \geq 1$.

Proposition 4.12 Let $D$ be an open set which is $\kappa$-fat at infinity. Then $\partial_{M}^{\infty} D$ consists of exactly one point.

Proof. Let $w \in \partial_{M}^{\infty} D$ and let $M_{D}(\cdot, w)$ be the corresponding Martin kernel. If the sequence $\left(y_{n}\right)_{n \geq 1} \subset D$ converges to $w$ in the Martin topology, then, by (M3)(a), $M_{D}\left(x, y_{n}\right)$ converge to $M_{D}(x, w)$. On the other hand, $\left|y_{n}\right| \rightarrow \infty$, thus by Theorem 4.9 ,

$$
\lim _{n \rightarrow \infty} M_{D}\left(x, y_{n}\right)=\lim _{\left|y_{n}\right| \rightarrow \infty} M_{D}\left(x, y_{n}\right)=M_{D}(x, \infty)
$$


Hence, for each $w \in \partial_{M}^{\infty} D$ it holds that $M_{D}(\cdot, w)=M_{D}(\cdot, \infty)$. Since, by (M3)(d), for two different Martin boundary points $w^{(1)}$ and $w^{(2)}$ it always holds that $M_{D}\left(\cdot, w^{(1)}\right) \neq M_{D}\left(\cdot, w^{(2)}\right)$, we conclude that the infinite part of the Martin boundary can be identified with the single point.

From now on we use the notation $\partial_{M}^{\infty} D=\left\{\partial_{\infty}\right\}$ and, for simplicity, we sometimes continue to write $M_{D}(x, \infty)$ for the more precise $M_{D}\left(x, \partial_{\infty}\right)$.

We now briefly discuss some properties of the finite part of the Martin boundary. Recall that $d$ denotes the Martin metric. For $\epsilon>0$ let

$$
K_{\epsilon}:=\left\{w \in \partial_{M}^{f} D: d\left(w, \partial_{\infty}\right) \geq \epsilon\right\}
$$

be a closed subset of $\partial_{M} D$. By the definition of the finite part of the Martin boundary, for each $w \in K_{\epsilon}$ there exists a bounded sequence $\left(y_{n}^{w}\right)_{n \geq 1} \subset D$ such that $\lim _{n \rightarrow \infty} d\left(y_{n}^{w}, w\right)=0$. Without loss of generality we may assume that $d\left(y_{n}^{w}, w\right)<\frac{\epsilon}{2}$ for all $n \geq 1$.

Lemma 4.13 There exists $C_{3}=C_{3}(\epsilon)>0$ such that $\left|y_{n}^{w}\right| \leq C_{3}$ for all $w \in K_{\epsilon}$ and all $n \geq 1$.

Proof. We first claim that for any sequence $\left(y_{n}\right)_{n \geq 1}$ of points in $D$, if $\left|y_{n}\right| \rightarrow \infty$, then $\lim _{n \rightarrow \infty} d\left(y_{n}, \partial_{\infty}\right)=$ 0, i.e., $\left(y_{n}\right)_{n \geq 1}$ converges to $\partial_{\infty}$ in the Martin topology. Indeed, since $D \cup \partial_{M} D$ is a compact metric space, $\left(y_{n}\right)$ has a convergent subsequence $\left(y_{n_{k}}\right)$. Let $w=\lim _{k \rightarrow \infty} y_{n_{k}}$ (in the Martin topology). Then $\lim _{k \rightarrow \infty} M_{D}\left(\cdot, y_{n_{k}}\right)=M_{D}(\cdot, w)$. On the other hand, from Theorem 4.9 and Proposition 4.12 we see that $\lim _{k \rightarrow \infty} M_{D}\left(\cdot, y_{n_{k}}\right)=M_{D}(\cdot, \infty)=M_{D}\left(\cdot, \partial_{\infty}\right)$. Therefore, $M_{D}(\cdot, w)=M_{D}\left(\cdot, \partial_{\infty}\right)$, which implies that $w=\partial_{\infty}$ by $(\mathrm{M} 3)(\mathrm{d})$. Since this argument also holds for any subsequence of $\left(y_{n}\right)_{n \geq 1}$, we conclude that $y_{n} \rightarrow \partial_{\infty}$ in the Martin topology.

Now suppose the lemma is not true. Then $\left\{y_{n}^{w}: w \in K_{\epsilon}, n \in \mathbb{N}\right\}$ contains a sequence $\left(y_{n_{k}}^{w_{k}}\right)_{k \geq 1}$ such that $\lim _{k \rightarrow \infty}\left|y_{n_{k}}^{w_{k}}\right|=\infty$. By the paragraph above, we have that $\lim _{k \rightarrow \infty} d\left(y_{n_{k}}^{w_{k}}, \partial_{\infty}\right)=0$. On the other hand,

$$
d\left(y_{n_{k}}^{w_{k}}, \partial_{\infty}\right) \geq d\left(w_{k}, \partial_{\infty}\right)-d\left(y_{n_{k}}^{w_{k}}, w_{k}\right) \geq \epsilon-\frac{\epsilon}{2}=\frac{\epsilon}{2} .
$$

This contradiction proves the claim.

Recall that an open set $D$ is called an exterior open set if $D^{c}$ is compact.

Corollary 4.14 If $D$ is an exterior open set, then $\partial_{\infty}$ is an isolated point of $\partial_{M} D$. Conversely, if $D$ is open and $\kappa$-fat at infinity, and $\partial_{\infty}$ is an isolated point of $\partial_{M} D$, then $D$ is an exterior open set.

Proof. Suppose that $D$ is an exterior open set. Then $D$ is $\kappa$-fat at infinity, hence $\partial_{M} D=$ $\partial_{M}^{f} D \cup\left\{\partial_{\infty}\right\}$. Since $D^{c}$ is compact we see that the Euclidean boundary $\partial D$ is bounded. We show that $\partial_{M}^{f} D$ is closed in the Martin topology. This will imply that $\left\{\partial_{\infty}\right\}$ is open in $\partial_{M} D$, hence isolated. Let $\left(w_{n}\right)_{n \geq 1}$ be a sequence in $\partial_{M}^{f} D$ which converges to $w \in \partial_{M} D$ in the Martin topology. For each $n \geq 1$, there exists a bounded sequence $\left(y_{k}^{w_{n}}\right)_{k \geq 1}$ such that $y_{k}^{w_{n}} \rightarrow w_{n}$ in the Martin 
topology. By Remark 4.11, we can assume that $\delta_{D}\left(y_{k}^{w_{n}}\right)=\delta_{\partial D}\left(y_{k}^{w_{n}}\right) \leq 1$ for all $n \geq 1, k \geq 1$. Since $\partial D$ is compact, the family $\left\{y_{k}^{w_{n}}: n \geq 1, k \geq 1\right\}$ is bounded. Further, because $\lim _{n \rightarrow \infty} d\left(w_{n}, w\right)=0$ and $\lim _{k \rightarrow \infty} d\left(y_{k}^{w_{n}}, w_{n}\right)=0$, we can find a sequence $\left(y_{k}\right)_{k \geq 1} \subset\left\{y_{k}^{w_{n}}: n \geq 1, k \geq 1\right\}$ such that $\lim _{k \rightarrow \infty} d\left(y_{k}, w\right)=0$. Clearly, the sequence $\left(y_{k}\right)_{k \geq 1}$ is bounded proving that $w \in \partial_{M}^{f} D$.

Conversely, assume that $\partial_{\infty}$ is an isolated point of $\partial_{M} D$. Then there exists $\epsilon>0$ such that $K_{\epsilon}=\left\{w \in \partial_{M}^{f} D: d\left(w, \partial_{\infty}\right) \geq \epsilon\right\}=\partial_{M}^{f} D$. Suppose that $D$ is not an exterior open set. Then both $D$ and $D^{c}$ are unbounded, and therefore $\partial D$ is unbounded as well. Hence, there exists $z \in \partial D$ such that $|z| \geq 3 C_{3}$ where $C_{3}=C_{3}(\epsilon)$ is the constant from Lemma 4.13, We can find a sequence $\left(z_{n}\right)_{n \geq 1} \subset D$ such that $z_{n} \rightarrow z$ (in the Euclidean topology) and $2 C_{3} \leq\left|z_{n}\right|$ for all $n \geq 1$. Since $D \cup \partial_{M} D$ is compact, there exist a subsequence $\left(z_{n_{k}}\right)_{k \geq 1}$ and $w \in D \cup \partial_{M} D$ such that $z_{n_{k}} \rightarrow w$ in the Martin topology. Clearly, $w \in \partial_{M} D$, and since $\left(z_{n_{k}}\right)$ is bounded, actually $w \in \partial_{M}^{f} D$. By Lemma 4.13, it holds that $\left|z_{n_{k}}\right| \leq C_{3}$ (for those $z_{n_{k}}$ for which $d\left(z_{n_{k}}, w\right) \leq \epsilon / 2$ ). But this contradicts that $\left|z_{n_{k}}\right| \geq 2 C_{3}$.

We continue by showing that $M_{D}\left(\cdot, \partial_{\infty}\right)$ is harmonic in $D$ with respect to $X$.

Lemma 4.15 For every bounded open $U \subset \bar{U} \subset D$ and every $x \in D, M_{D}\left(X_{\tau_{U}}, \partial_{\infty}\right)$ is $\mathbb{P}_{x^{-}}$ integrable.

Proof. Let $\left(y_{m}\right)_{m \geq 1}$ be a sequence in $D \backslash \bar{U}$ such that $\left|y_{m}\right| \rightarrow \infty$. Then $M_{D}\left(\cdot, y_{m}\right)$ is regular harmonic in $U$. Hence, by Fatou's lemma,

$$
\begin{aligned}
& \mathbb{E}_{x}\left[M_{D}\left(X_{\tau_{U}}, \partial_{\infty}\right)\right]=\mathbb{E}_{x}\left[\lim _{m \rightarrow \infty} M_{D}\left(X_{\tau_{U}}, y_{m}\right)\right] \leq \liminf _{m \rightarrow \infty} \mathbb{E}_{x}\left[M_{D}\left(X_{\tau_{U}}, y_{m}\right)\right] \\
& =\liminf _{m \rightarrow \infty} M_{D}\left(x, y_{m}\right)=M_{D}\left(x, \partial_{\infty}\right)<\infty
\end{aligned}
$$

Lemma 4.16 For each $x \in D$ and $\rho \in\left(0, \frac{1}{3} \delta_{D}(x)\right]$,

$$
M_{D}\left(x, \partial_{\infty}\right)=\mathbb{E}_{x}\left[M_{D}\left(X_{\tau_{B(x, \rho)}}, \partial_{\infty}\right)\right]
$$

Proof. Fix $x \in D$ and $\rho \in\left(0, \frac{1}{3} \delta_{D}(x)\right]$. For $m \in \mathbb{N}$, let $\eta_{m}:=(\kappa / 2)^{-m} \rho$. Let $\widetilde{m} \in \mathbb{N}$ be large enough so that $\eta_{\widetilde{m}} \geq(2|x|+2 \rho) \vee R$. In case $m \geq \widetilde{m}$, let $A_{m}:=A_{\eta_{m}}$. Then for $m \geq \widetilde{m}, M_{D}\left(\cdot, A_{m}\right)$ is regular harmonic in $D \backslash B\left(A_{m}, \kappa \eta_{m}\right)$ and $B(x, \rho) \subset D \backslash B\left(A_{m}, \kappa \eta_{m}\right)$, hence

$$
M_{D}\left(x, A_{m}\right)=\mathbb{E}_{x}\left[M_{D}\left(X_{\tau_{B(x, \rho)}}, A_{m}\right)\right], \quad m \geq \widetilde{m} .
$$

From now on we assume that $m \geq \widetilde{m}$. To prove the statement of the lemma it suffices to show that there exists $m_{1} \in \mathbb{N}, m_{1} \geq \widetilde{m}$, such that the family $\left\{M_{D}\left(X_{\tau_{B(x, \rho)}}, A_{m}\right): m \geq m_{1}\right\}$ is uniformly integrable with respect to $\mathbb{P}_{x}$. This will allow us to exchange the order of the expectation and the limit when we take the limit $m \rightarrow \infty$ in (4.10), thus proving the statement. 
Choose $\widetilde{m}$ even larger so that $(\kappa / 2)^{-\widetilde{m}} \rho \geq 2\left|x_{0}\right|$, and let $m \geq \widetilde{m}$. Let $w \in D \cap B\left(0, \eta_{m}\right)$. Then $G_{D}(w, \cdot)$ is regular harmonic in $D \cap \bar{B}\left(0, \eta_{m}\right)^{c}$ and vanishes on $D^{c} \cap \bar{B}\left(0, \eta_{m}\right)^{c}$. The same is valid for $G_{D}\left(x_{0}, \cdot\right)$. Since $A_{m} \in D \cap \bar{B}\left(0,(\kappa+1) \eta_{m}\right)^{c}$, Corollary 1.2 implies that for $w \in D \cap B\left(0, \eta_{m}\right)$,

$$
M_{D}\left(w, A_{m}\right)=\frac{G_{D}\left(w, A_{m}\right)}{G_{D}\left(x_{0}, A_{m}\right)} \leq C_{2} \frac{G_{D}(w, y)}{G_{D}\left(x_{0}, y\right)}=C_{2} M_{D}(w, y), \quad \text { for all } y \in D \cap \bar{B}\left(0,(\kappa+1) \eta_{m}\right)^{c} .
$$

Hence, by letting $|y| \rightarrow \infty$,

$$
M_{D}\left(w, A_{m}\right) \leq C_{2} M_{D}\left(w, \partial_{\infty}\right), \quad m \geq \widetilde{m}
$$

Let $\epsilon>0$ be arbitrary. By Lemma 4.15] and (4.12), there exists $N_{0}>0$ such that

$$
\begin{aligned}
& \mathbb{E}_{x}\left[M_{D}\left(X_{\tau_{B(x, \rho)}}, A_{m}\right): X_{\tau_{B(x, \rho)}} \in D \cap B\left(0, \eta_{m}\right), M_{D}\left(X_{\tau_{B(x, \rho)}}, A_{m}\right)>N_{0}\right] \\
& \quad \leq C_{2} \mathbb{E}_{x}\left[M_{D}\left(X_{\tau_{B(x, \rho)}}, \partial_{\infty}\right): C_{2} M_{D}\left(X_{\tau_{B(x, \rho)}}, \partial_{\infty}\right)>N_{0}\right] \leq C_{2} \frac{\epsilon}{2 C_{2}}=\frac{\epsilon}{2} .
\end{aligned}
$$

On the other hand,

$$
\begin{aligned}
\mathbb{E}_{x} & {\left[M_{D}\left(X_{\tau_{B(x, \rho)}}, A_{m}\right): X_{\tau_{B(x, \rho)}} \in D \cap \bar{B}\left(0, \eta_{m}\right)^{c}\right] } \\
& =\int_{D \cap \bar{B}\left(0, \eta_{m}\right)^{c}} M_{D}\left(v, A_{m}\right) K_{B(x, \rho)}(x, v) d v \\
& \leq c_{1} \int_{D \cap \bar{B}\left(0, \eta_{m}\right)^{c}} M_{D}\left(v, A_{m}\right) j(|v-x|-\rho) \phi\left(\rho^{-2}\right)^{-1} d v,
\end{aligned}
$$

where the last inequality follows from the uniform upper estimate of the Poisson kernel in (2.14). Choose $m_{0} \geq \widetilde{m}$ large enough such that for $m \geq m_{0}$ and $v \in D \cap \bar{B}\left(0, \eta_{m}\right)^{c}$ it holds that $|v-x|-\rho \geq$ $|v| / 2$. Then $j(|v-x|-r) \leq j(|v| / 2) \leq c_{2} j(|v|)$ by (2.10). Hence, by treating $\phi\left(\rho^{-2}\right)^{-1}$ as a constant (depending on $\rho$, but note that $\rho$ is fixed), we get that

$$
\begin{aligned}
\mathbb{E}_{x} & {\left[M_{D}\left(X_{\tau_{B(x, \rho)}}, A_{m}\right): X_{\tau_{B(x, \rho)}} \in D \cap \bar{B}\left(0, \eta_{m}\right)^{c}\right] } \\
\leq & c_{3} \int_{D \cap \bar{B}\left(0, \eta_{m}\right)^{c}} M_{D}\left(v, A_{m}\right) j(|v|) d v, \\
& =c_{3} G_{D}\left(x_{0}, A_{m}\right)^{-1} \int_{D \cap \bar{B}\left(0, \eta_{m}\right)^{c}} G_{D}\left(v, A_{m}\right) j(|v|) d v, \quad m \geq m_{0} .
\end{aligned}
$$

By Lemma 4.2 (applied to $r=\eta_{m_{0}}$ ) we have that

$$
G_{D}\left(x_{0}, A_{m}\right)^{-1} \leq c_{4}(\kappa / 2)^{(-d+\gamma)\left(m-m_{0}\right)} G_{D}\left(x_{0}, A_{m_{0}}\right)^{-1}, \quad m \geq m_{0},
$$

where $\gamma \in(0, d)$. Now we estimate the integral in (4.15):

$$
\begin{aligned}
& \int_{D \cap \bar{B}\left(0, \eta_{m}\right)^{c}} G_{D}\left(v, A_{m}\right) j(|v|) d v \\
& \quad \leq \int_{D \cap B\left(A_{m},(\kappa+1 / 2) \eta_{m}\right)} G\left(v, A_{m}\right) j(|v|) d v+\int_{D \cap B\left(A_{m},(\kappa+1 / 2) \eta_{m}\right)^{c} \cap \bar{B}\left(0, \eta_{m}\right)^{c}} G_{D}\left(v, A_{m}\right) j(|v|) d v \\
& \quad=: \quad I_{1}+I_{2} .
\end{aligned}
$$


To estimate $I_{1}$, note that if $v \in B\left(A_{m},(\kappa+1 / 2) \eta_{m}\right)$, then $|v| \geq\left|A_{m}\right|-\left|v-A_{m}\right| \geq(\kappa+1) \eta_{m}-$ $(\kappa+1 / 2) \eta_{m}=(1 / 2) \eta_{m}=(1 / 2)(\kappa / 2)^{-m} \rho$, hence $j(|v|) \leq c_{5} j\left((\kappa / 2)^{-m} \rho\right)$ by (2.10). Therefore, by Theorem 2.3 and (2.3)

$$
\begin{aligned}
I_{1} & \leq c_{6} j\left((\kappa / 2)^{-m} \rho\right) \int_{B\left(A_{m},(\kappa / 2+1) \eta_{m}\right)} \frac{1}{\left|v-A_{m}\right|^{d} \phi\left(\left|v-A_{m}\right|^{-2}\right)} d v \\
& \leq c_{7} j\left((\kappa / 2)^{-m} \rho\right) \int_{0}^{(\kappa / 2+1) \eta_{m}} \frac{1}{s \phi\left(s^{-2}\right)} d s \\
& \leq c_{8} j\left((\kappa / 2)^{-m} \rho\right) \phi\left(\left((\kappa / 2)^{-m} \rho\right)^{-2}\right)^{-1} \leq c_{9}\left((\kappa / 2)^{-m} \rho\right)^{-d} .
\end{aligned}
$$

In order to estimate $I_{2}$, let $v \in D \cap B\left(A_{m},(\kappa+1 / 2) \eta_{m}\right)^{c} \cap \bar{B}\left(0, \eta_{m}\right)^{c}$. If $|v| \geq \kappa^{-1}(1-\kappa)^{-1} \eta_{m}$, then $\left|v-A_{m}\right| \geq|v|-\left|A_{m}\right| \geq \kappa|v|$. If $\eta_{m} \leq|v|<\kappa^{-1}(1-\kappa)^{-1} \eta_{m}$, then $\left|v-A_{m}\right| \geq(\kappa+1 / 2) \eta_{m} \geq$ $\kappa(1-\kappa)(\kappa+1 / 2)|v|$. Thus, in any case, $G_{D}\left(v, A_{m}\right) \leq g\left(\left|v-A_{m}\right|\right) \leq c_{10} g(|v|)$ by (3.1). Therefore, by Theorem 2.3 ,

$$
\begin{aligned}
I_{2} & \leq c_{10} \int_{D \cap B\left(A_{m},(\kappa+1 / 2) \eta_{m}\right)^{c} \cap \bar{B}\left(0, \eta_{m}\right)^{c}} g(|v|) j(|v|) d v \\
& \leq c_{10} \int_{\bar{B}\left(0, \eta_{m}\right)^{c}} g(|v|) j(|v|) d v \leq c_{11} \int_{\eta_{m}}^{\infty} \frac{1}{s^{d+1}} d s=c_{12} \eta_{m}^{-d}=c_{12}\left((\kappa / 2)^{-m} \rho\right)^{-d} .
\end{aligned}
$$

Hence,

$$
\int_{D \cap \bar{B}\left(0, \eta_{m}\right)^{c}} G_{D}\left(v, A_{m}\right) j(|v|) d v \leq c_{13}\left((\kappa / 2)^{-m} \rho\right)^{-d}, \quad m \geq m_{0} .
$$

By combining (4.14)-(4.17) we get that

$$
\begin{aligned}
& \mathbb{E}_{x}\left[M_{D}\left(X_{\tau_{B(x, \rho)}}, A_{m}\right): X_{\tau_{B(x, \rho)}} \in D \cap \bar{B}\left(0, \eta_{m}\right)^{c}\right] \\
& \quad \leq c_{14}(\kappa / 2)^{(-d+\gamma)\left(m-m_{0}\right)} G_{D}\left(x_{0}, A_{m_{0}}\right)^{-1}\left((\kappa / 2)^{-m} \rho\right)^{-d} \\
& \quad \leq c_{15} G_{D}\left(x_{0}, A_{m_{0}}\right)^{-1}(\kappa / 2)^{d m_{0}}(\kappa / 2)^{\gamma\left(m-m_{0}\right)}
\end{aligned}
$$

where in the last line we treat $\rho$ as a constant. Since $\gamma>0$, we can choose $m_{1}=m_{1}\left(\epsilon, m_{0}, d, \phi, \rho\right)>$ $m_{0}$ large enough so that the right-hand side in (4.18) is less than $\epsilon / 2$ for all $m \geq m_{1}$.

Finally, for $m \geq m_{1}$ we have

$$
\begin{aligned}
\mathbb{E}_{x}[ & \left.M_{D}\left(X_{\tau_{B(x, \rho)}}, A_{m}\right): M_{D}\left(X_{\tau_{B(x, \rho)}}, A_{m}\right)>N_{0}\right] \\
\leq & \mathbb{E}_{x}\left[M_{D}\left(X_{\tau_{B(x, \rho)}}, A_{m}\right): X_{\tau_{B(x, \rho)}} \in D \cap \bar{B}\left(0, \eta_{m}\right)^{c}\right] \\
& \quad+\mathbb{E}_{x}\left[M_{D}\left(X_{\tau_{B(x, \rho)}}, A_{m}\right): X_{\tau_{B(x, \rho)}} \in D \cap B\left(0, \eta_{m}\right), M_{D}\left(X_{\tau_{B(x, \rho)}}, A_{m}\right)>N_{0}\right] \\
\leq & \frac{\epsilon}{2}+\frac{\epsilon}{2}=\epsilon .
\end{aligned}
$$

Hence $\left\{M_{D}\left(X_{\tau_{B(x, \rho)}}, A_{m}\right): m \geq m_{1}\right\}$ is uniformly integrable with respect to $\mathbb{P}_{x}$.

Theorem 4.17 The function $M_{D}\left(\cdot, \partial_{\infty}\right)$ is harmonic in $D$ with respect to $X$. 
Proof. The proof of the theorem is exactly the same as that of [12, Theorem 3.9].

Let $x \in D$ and choose $r \geq\left(2|x| \vee\left|x_{0}\right|\right)$. By Corollary 1.2 we have that for all $y \in D \cap \bar{B}(0, r)^{c}$

$$
\frac{G_{D}(x, y)}{G_{D}\left(x_{0}, y\right)} \leq C_{2} \frac{G_{D}\left(x, A_{r}\right)}{G_{D}\left(x_{0}, A_{r}\right)} .
$$

By letting $|y| \rightarrow \infty$ we get that

$$
M_{D}\left(x, \partial_{\infty}\right) \leq C_{2} \frac{G_{D}\left(x, A_{r}\right)}{G_{D}\left(x_{0}, A_{r}\right)} .
$$

Suppose that $z \in \partial D$ is a regular boundary point. Then $\lim _{x \rightarrow z} G_{D}\left(x, A_{r}\right)=0$ implying also that

$$
\lim _{x \rightarrow z} M_{D}\left(x, \partial_{\infty}\right)=0, \quad \text { for every regular boundary point } z \in \partial D .
$$

Lemma 4.18 Suppose that $u$ is a bounded nonnegative harmonic function for $X^{D}$. If there exists a polar set $N \subset \partial D$ such that for any $z \in \partial D \backslash N$

$$
\lim _{D \ni x \rightarrow z} u(x)=0
$$

and

$$
\lim _{x \in D,|x| \rightarrow \infty} u(x)=0
$$

then $u$ is identically equal to zero.

Proof. Take an increasing sequence of bounded open sets $\left\{D_{n}\right\}_{n \geq 1}$ satisfying $\overline{D_{n}} \subset D_{n+1}$ and $\cup_{n=1}^{\infty} D_{n}=D$. Then $\lim _{n \rightarrow \infty} \tau_{D_{n}}=\tau_{D}$ and by the quasi-left continuity, $\lim _{n \rightarrow \infty} X_{\tau_{D_{n}}}=X_{\tau_{D}}$ if $\tau_{D}<\infty$, and $\lim _{n \rightarrow \infty}\left|X_{\tau_{D_{n}}}\right|=\infty$ if $\tau_{D}=\infty$.

Since $N$ is polar, we have $\mathbb{P}_{x}\left(X_{\tau_{D}} \in N, \tau_{D}<\infty\right)=0, x \in D$. By harmonicity we have for every $x \in D$ and all $n \geq 1$

$$
\begin{aligned}
u(x)= & \mathbb{E}_{x}\left[u\left(X_{\tau_{D_{n}}}\right)\right] \\
= & \mathbb{E}_{x}\left[u\left(X_{\tau_{D_{n}}}\right), \tau_{D}=\infty\right]+\mathbb{E}_{x}\left[u\left(X_{\tau_{D_{n}}}\right), \tau_{D_{m}}=\tau_{D} \text { for some } m \geq 1\right] \\
& +\mathbb{E}_{x}\left[u\left(X_{\tau_{D_{n}}}\right), \tau_{D_{m}}<\tau_{D}<\infty \text { for all } m \geq 1\right] .
\end{aligned}
$$

By using bounded convergence theorem we get that

$$
\lim _{n \rightarrow \infty} \mathbb{E}_{x}\left[u\left(X_{\tau_{D_{n}}}\right), \tau_{D}=\infty\right]=\mathbb{E}_{x}\left[\lim _{n \rightarrow \infty} u\left(X_{\tau_{D_{n}}}\right), \tau_{D}=\infty\right]=0,
$$

since $\left|X_{\tau_{D_{n}}}\right| \rightarrow \infty$ on $\left\{\tau_{D}=\infty\right\}$. Next, since $u=0$ on $D^{c}$,

$$
\begin{aligned}
\lim _{n \rightarrow \infty} & \mathbb{E}_{x}\left[u\left(X_{\tau_{D_{n}}}\right), \tau_{D_{m}}=\tau_{D} \text { for some } m \geq 1\right] \\
\quad= & \mathbb{E}_{x}\left[\lim _{n \rightarrow \infty} u\left(X_{\tau_{D_{n}}}\right), \tau_{D_{m}}=\tau_{D} \text { for some } m \geq 1\right] \\
\quad= & \mathbb{E}_{x}\left[u\left(X_{\tau_{D}}\right), \tau_{D_{m}}=\tau_{D} \text { for some } m \geq 1\right]=0 .
\end{aligned}
$$


Finally, if $\tau_{D_{m}}<\tau_{D}<\infty$, then $\lim _{n \rightarrow \infty} X_{\tau_{D_{n}}} \in \partial D \backslash N \mathbb{P}_{x^{-}}$a.s. Hence

$$
\begin{aligned}
\lim _{n \rightarrow \infty} & \mathbb{E}_{x}\left[u\left(X_{\tau_{D_{n}}}\right), \tau_{D_{m}}<\tau_{D}<\infty \text { for all } m \geq 1\right] \\
\quad= & \mathbb{E}_{x}\left[\lim _{n \rightarrow \infty} u\left(X_{\tau_{D_{n}}}\right) \mathbf{1}_{\left\{X_{\tau_{D}} \in \partial D \backslash N\right\}}, \tau_{D_{m}}<\tau_{D}<\infty \text { for all } m \geq 1\right]=0 .
\end{aligned}
$$

Therefore, $u(x)=0$ for every $x \in D$.

Recall that a positive harmonic function $f$ for $X^{D}$ is minimal if, whenever $g$ is a positive harmonic function for $X^{D}$ with $g \leq f$ on $D$, one must have $f=c g$ for some constant $c$.

Proof of Theorem 1.4. It remains to show that $\partial_{\infty}$ is a minimal boundary point, i.e., that $M_{D}\left(\cdot, \partial_{\infty}\right)$ is a minimal harmonic function.

Let $h$ be a positive harmonic function for $X^{D}$ such that $h \leq M_{D}\left(\cdot, \partial_{\infty}\right)$. By the Martin representation in [16], there is a measure on $\partial_{M} D=\partial_{M}^{f} D \cup\left\{\partial_{\infty}\right\}$ such that

$$
h(x)=\int_{\partial_{M} D} M_{D}(x, w) \mu(d w)=\int_{\partial_{M}^{f} D} M_{D}(x, w) \mu(d w)+M_{D}\left(x, \partial_{\infty}\right) \mu\left(\left\{\partial_{\infty}\right\}\right) .
$$

In particular, $h\left(x_{0}\right)=\mu\left(\partial_{M} D\right) \leq M\left(x_{0}, \partial_{\infty}\right)=1$ (because of the normalization at $\left.x_{0}\right)$. Hence, $\mu$ is a sub-probability measure.

For $\epsilon>0, K_{\epsilon}$ is the compact subset of $\partial_{M} D$ defined in (4.9). Define

$$
u(x):=\int_{K_{\epsilon}} M_{D}(x, w) \mu(d w) .
$$

Then $u$ is a positive harmonic function with respect to $X^{D}$ and bounded above as

$$
u(x)=h(x)-\mu(\{\infty\}) M_{D}\left(x, \partial_{\infty}\right) \leq(1-\mu(\{\infty\})) M_{D}\left(x, \partial_{\infty}\right) .
$$

We claim that $\lim _{|x| \rightarrow \infty} u(x)=0$. By Lemma 4.13 there exists $C_{3}=C_{3}(\epsilon)>0$ such that for each $w \in K_{\epsilon}$ there exists a sequence $\left(y_{n}^{w}\right)_{n \geq 1} \subset D$ converging to $w$ in the Martin topology and satisfying $\left|y_{n}^{w}\right| \leq C_{3}$. Without loss of generality we may assume that $C_{3} \geq R$. Fix a point $x_{1} \in D \cap \bar{B}\left(0,2 C_{3}\right)^{c}$ and choose an arbitrary point $y_{0} \in D \cap B\left(0, C_{3}\right)$. Then for any $x \in D \cap \bar{B}\left(0,2 C_{3}\right)^{c}$ and any $y \in D \cap B\left(0, C_{3}\right)$ we have that

$$
\frac{G_{D}(x, y)}{G_{D}\left(x_{0}, y\right)}=\frac{G_{D}(x, y)}{G_{D}\left(x_{1}, y\right)} \frac{G_{D}\left(x_{1}, y\right)}{G_{D}\left(x_{0}, y\right)} \leq c_{1} \frac{G_{D}\left(x, y_{0}\right)}{G_{D}\left(x_{1}, y_{0}\right)} \frac{G_{D}\left(x_{1}, y\right)}{G_{D}\left(x_{0}, y\right)} \leq c_{1} \frac{G\left(x, y_{0}\right)}{G_{D}\left(x_{1}, y_{0}\right)} \frac{G_{D}\left(x_{1}, y\right)}{G_{D}\left(x_{0}, y\right)},
$$

where the first inequality follows from the boundary Harnack principle, Theorem 2.8. Therefore for each $w \in K_{\epsilon}$ we have

$$
\begin{aligned}
M_{D}(x, w) & =\lim _{n \rightarrow \infty} \frac{G_{D}\left(x, y_{n}^{w}\right)}{G_{D}\left(x_{0}, y_{n}^{w}\right)} \leq c_{1} \frac{G\left(x, y_{0}\right)}{G_{D}\left(x_{1}, y_{0}\right)} \lim _{n \rightarrow \infty} \frac{G_{D}\left(x_{1}, y_{n}^{w}\right)}{G_{D}\left(x_{0}, y_{n}^{w}\right)} \\
& =c_{1} \frac{G\left(x, y_{0}\right)}{G_{D}\left(x_{1}, y_{0}\right)} M_{D}\left(x_{1}, w\right) \leq c_{1} \frac{G\left(x, y_{0}\right)}{G_{D}\left(x_{1}, y_{0}\right)} \sup _{w \in K_{\epsilon}} M_{D}\left(x_{1}, w\right)=c_{2} G\left(x, y_{0}\right)
\end{aligned}
$$


by continuity of the Martin kernel (M3)(c). Now we let $|x| \rightarrow \infty$ and use that $G\left(x, y_{0}\right) \rightarrow 0$ to conclude that $\lim _{|x| \rightarrow \infty, x \in D} M_{D}(x, w)=0$ uniformly for $w \in K_{\epsilon}$. By Theorem 4.9, this and (4.20) immediately imply that $\lim _{|x| \rightarrow \infty} u(x)=0$.

From (4.19) we see that $\lim _{x \rightarrow z} u(x)=0$ for every regular $z \in \partial D$. Since the set of irregular boundary points is polar (cf. [1, (VI.4.6), (VI.4.10)]), Lemma 4.18 implies that $u \equiv 0$. This means that $\nu=\mu_{\mid K_{\epsilon}}=0$. Since $\epsilon>0$ was arbitrary and $\partial_{M}^{f} D=\cup_{\epsilon>0} K_{\epsilon}$, we see that $\mu_{\mid \partial_{M}^{f} D}=0$. Hence $h=\mu\left(\left\{\partial_{\infty}\right\}\right) M\left(\cdot, \partial_{\infty}\right)$ showing that $M\left(\cdot, \partial_{\infty}\right)$ is minimal. Therefore we have proved Theorem 1.4

At the end we briefly discuss the Martin boundary of the half-space $\mathbb{H}=\left\{x=\left(\widetilde{x}, x_{d}\right): \widetilde{x} \in\right.$ $\left.\mathbb{R}^{d-1}, x_{d}>0\right\}$. Let $V(r)$ be the renewal function of the ladder height process of one-dimensional subordinate Brownian motion $X_{t}^{d}=W^{d}\left(S_{t}\right)$. It is known that the function $w(x):=V\left(\left(x_{d}\right)^{+}\right)$is harmonic in $\mathbb{H}$ with respect to $X$ (see [11]). Moreover, for every $z \in \partial \mathbb{H}:=\left\{x=\left(\widetilde{x}, x_{d}\right): \widetilde{x} \in\right.$ $\left.\mathbb{R}^{d-1}, x_{d}=0\right\}$ it holds that $\lim _{x \rightarrow z} w(x)=0$. Therefore we can conclude that $w$ is proportional to the minimal harmonic function $M_{\mathbb{H}}(\cdot, \infty)$. In the next corollary we compute the full Martin boundary of $\mathbb{H}$.

Corollary 4.19 The Martin boundary and the minimal Martin boundary of the half space $\mathbb{H}$ with respect to $X$ can be identified with $\partial \mathbb{H} \cup\{\infty\}$ and $M_{\mathbb{H}}(x, \infty)=w(x) / w\left(x_{0}\right)$ for $x \in \mathbb{H}$.

Proof. By Theorem 1.4 and the argument before the statement of this corollary, we only need to show that the finite part $\partial_{M}^{f} \mathbb{H}$ of the Martin boundary of $\mathbb{H}$ can be identified with the Euclidean boundary $\partial \mathbb{H}$ and that all points are minimal. This was shown in [12, Theorem 3.13] under the assumption that $\phi$ is comparable to the regularly varying function at infinity. Even though this assumption is stronger than (H1), using results in this paper and [15] (instead of using properties of regularly varying function) one can follow the same proof line by line and show that under the assumption (H1) and $d>2 \delta_{2}$, the finite part of Martin boundary $\partial_{M}^{f} \mathbb{H}$ can be identified with the Euclidean boundary $\partial \mathbb{H}$ and that all points are minimal. We omit the details.

\section{References}

[1] R. M. Blumenthal and R. K. Getoor: Markov Processes and Potential Theory, Academic Press, New York, 1968.

[2] K. Bogdan: The boundary Harnack principle for the fractional Laplacian. Studia Math. 123(1)(1997), $43-80$.

[3] K. Bogdan, T. Byczkowski, T. Kulczycki, M. Ryznar, R. Song, and Z. Vondraček: Potential analysis of stable processes and its extensions. Lecture Notes in Mathematics, 1980. Springer-Verlag, Berlin, 2009.

[4] K. Bogdan, T. Kulczycki and M. Kwaśnicki: Estimates and structure of $\alpha$-harmonic functions. Probab. Th. Rel. Fields, 140 (2008), 345-381. 
[5] K. Bogdan, T. Kumagaii and M. Kwaśnicki: Boundary Harnack inequality for Markov processes with jumps. Preprint. arXiv:1207.3160.

[6] E. B. Dynkin: Markov processes, Vol. I. Academic Press, New York, 1965

[7] N. Ikeda and S. Watanabe: On some relations between the harmonic measure and the Lévy measure for a certain class of Markov processes, J. Math. Kyoto Univ. 2 (1962), 79-95.

[8] P. Kim and A. Mimica: Harnack inequalities for subordinate Brownian motions, Electron. J. Probab. 17 (2012), \#37.

[9] P. Kim and A. Mimica: Green function estimates for subordinate Brownian motions: stable and beyond, Trans. Amer. Math. Soc., to appear (2012)

[10] P. Kim, R. Song and Z. Vondraček: Boundary Harnack principle for subordinate Brownian motion. Stoch. Proc. Appl. 119 (2009), 1601-1631.

[11] P. Kim, R. Song and Z. Vondraček: Potential theory of subordinated Brownian motions revisited. Stochastic analysis and applications to finance, essays in honour of Jia-an Yan. Interdisciplinary Mathematical Sciences - Vol. 13, World Scientific, 2012, pp. 243-290.

[12] P. Kim, R. Song and Z. Vondraček: Minimal thinness for subordinate Brownian motion in half space. Ann. Inst. Fourier 62 (3) (2012), 1045-1080.

[13] P. Kim, R. Song and Z. Vondraček: Uniform boundary Harnack principle for rotationally symmetric Lévy processes in general open sets, Sci. China Math. 55, (2012), 2193-2416.

[14] P. Kim, R. Song and Z. Vondraček: Potential theory of subordinate Brownian motions with Gaussian components. Stoch. Proc. Appl. 123(3) (2013) 764-795.

[15] P. Kim, R. Song and Z. Vondraček: Global uniform boundary Harnack principle with explicit decay rate and its application. Preprint, 2012.

[16] H. Kunita and T. Watanabe: Markov processes and Martin boundaries I, Illinois J. Math. 9(3) (1965) $485-526$.

[17] M. Kwaśnicki: Intrinsic ultracontractivity for stable semigroups on unbounded open sets. Potential Anal. 31 (2009), 57-77.

[18] P. W. Millar: First passage distributions of processes with independent increments. Ann. Probab., 3 (1975), 215-233.

[19] K.-I. Sato: Lévy Processes and Infinitely Divisible Distributions. Cambridge University Press, Cambridge, 1999.

[20] R. L. Schilling, R. Song and Z. Vondraček: Bernstein Functions: Theory and Applications. de Gruyter Studies in Mathematics 37. Berlin: Walter de Gruyter, 2010.

[21] M. L. Silverstein: Classification of coharmonic and coinvariant functions for a Lévy process. Ann. Probab. 8 (1980), 539-575.

[22] R. Song and J. Wu: Boundary Harnack principle for symmetric stable processes. J. Funct. Anal. 168(2) (1999), 403-427.

[23] P. Sztonyk: On harmonic measure for Lévy processes. Probab. Math. Statist., 20 (2000), 383-390.

\section{Panki Kim}

Department of Mathematical Sciences and Research Institute of Mathematics, 
Seoul National University, Building 27, 1 Gwanak-ro, Gwanak-gu Seoul 151-747, Republic of Korea

E-mail: pkim@snu.ac.kr

Renming Song

Department of Mathematics, University of Illinois, Urbana, IL 61801, USA

E-mail: rsong@math.uiuc.edu

\section{Zoran Vondraček}

Department of Mathematics, University of Zagreb, Zagreb, Croatia

Email: vondra@math.hr 